\title{
Exact Schauder estimates of solutions of a boundary value problem for parabolic second order equations and their application to a nonlinear problem
}

\author{
by Mikhail Borsuk (Olsztyn)
}

\begin{abstract}
We establish exact Schauder estimates of solutions of the transmission problem for linear parabolic second order equations with explicit dependence on the smoothness of the coefficients. Next we apply the estimates to the solvability of the nonlinear transmission problem.
\end{abstract}

Introduction. In order to investigate the solvability of nonlinear boundary value problems for elliptic or parabolic equations it is necessary to look closer at the solutions of linear problems and obtain sharper estimates for these solutions. Such estimates are said to be of Schauder type. However it has turned out that usual estimates of Schauder type are sufficient only for the proof of solvability for the Dirichlet problem as well as for problems with semilinear boundary conditions. For other boundary value problems, when the boundary conditions contain strong nonlinearities, it is essential to derive exact a priori estimates of Schauder type for solutions of the corresponding linear problem with explicit dependence on the smoothness of the coefficients of the linear problems (see [5, Chapt. X]). Such an estimate was derived apparently for the first time by R. Caccioppoli and J. Schauder (1934, see [7, Chapt. V]) for elliptic equations.

An exact a priori estimate of Schauder type for solutions of the linear oblique derivative problem for elliptic second order equations with smooth coefficients was derived by R. Fiorenza [3] and with discontinuous coefficients by the author [1]. For parabolic equations only usual estimates of Schauder type have been studied: in [4] for oblique derivative problems, in [6] for general boundary value problems with smooth coefficients, and in [8] for discontinuous coefficients. An exact a priori estimate (in the above-mentioned sense) for solutions of a parabolic boundary value problem for second order

2000 Mathematics Subject Classification: 35J65, 35J70, 35B45.

Key words and phrases: Schauder estimate, transmission problem, quasilinear problem. 
equations is proved here for the first time. The results of this paper were announced without proofs in [2].

This paper consists of four sections. In Section 1 we collect some basic definitions, notations, preliminaries and state our main results. Boundary value problems are reduced to problems with zero initial data in Section 2. Then we derive exact a priori local estimates of Schauder type (in Section 3 we give detailed estimates only near the interface). The explicit dependence on the smoothness of coefficients of the linear problem in the exact Schauder estimates enables us to prove in Section 4 a conditional existence theorem for the general quasilinear problem in a composite domain. This theorem states essentially that the general quasilinear problem is classically solvable if there exist a priori estimates for its solutions in the space $C^{(1, \lambda)}$.

\section{Preliminaries}

1.1. Notations. We introduce the following notations and definitions:

- $E_{m}$ : the $m$-dimensional euclidean space of points $x=\left(x^{\prime}, x^{m}\right), x^{\prime}=$ $\left(x^{1}, \ldots, x^{m-1}\right)$;

- $|x-y|=\left(\sum_{i=1}^{m}\left(x^{i}-y^{i}\right)^{2}\right)^{1 / 2}$;

- $E_{m+1}=E_{m} \times(-\infty, \infty)$ : the $(m+1)$-dimensional euclidean space of points $(x, t)$;

- $\sqrt{|x-y|^{2}+|t-\tau|}$ : "parabolic" distance between two points $(x, t)$ and $(y, \tau)$ of $E_{m+1}$;

- $\Omega \subset E_{m}$ : a bounded domain with boundary $\partial \Omega$;

- $\gamma$ : the union of disjoint $(m-1)$-dimensional surfaces lying in the interior of $\Omega$ and dividing it into a finite number of domains: $\Omega=\bigcup_{q=1}^{N} \Omega^{(q)}$, where the surfaces forming $\gamma$ have no common points and do not intersect $\partial \Omega$; without loss of generality we assume $N=2$ so that always $q=1,2$; we set

$$
r(\gamma, \partial \Omega) \equiv \inf _{x \in \gamma, y \in \partial \Omega}|x-y|=r_{0}
$$

- $\Omega^{(1)}$ : a domain lying inside of $\gamma$;

- $\bar{\Omega}^{(1)}=\Omega^{(1)} \cup \gamma, \Omega^{(2)}=\Omega \backslash\left(\Omega^{(1)} \cup \gamma\right), \bar{\Omega}^{(2)}=\Omega^{(2)} \cup \gamma \cup \partial \Omega$;

- $\Gamma_{m}(x, \varrho)$ : the $m$-dimensional ball in $E_{m}$ of radius $\varrho$ with centre at $x$;

- $Q_{T}=\Omega \times(0, T)$ : a cylinder;

- $Q_{T}^{(q)}=\Omega^{(q)} \times(0, T)$;

- $Q_{t_{1}, t_{2}}=\Omega \times\left(t_{1}, t_{2}\right)$;

- $Q_{t_{1}, t_{2}}^{(q)}=\Omega^{(q)} \times\left(t_{1}, t_{2}\right)$;

- $\bar{\Omega}=\Omega \cup \partial \Omega$;

- $\bar{\Omega}_{T}=\bar{\Omega} \times[0, T]$;

- $\bar{Q}_{T}^{(q)}=\bar{\Omega}^{(q)} \times[0, T]$

- $\gamma_{T}=\gamma \times(0, T]$ : the lateral surface of the cylinder $Q_{T}^{(1)}$; 
- $\partial_{T} \Omega=\partial \Omega \times(0, T]$ : the lateral surface of the cylinder $Q_{T}$;

- $\Pi=\left\{(y, t) \in E_{m+1} \mid y_{m}=0\right\} \subset E_{m+1}$

- $\Pi_{T}=\left\{(y, t) \in E_{m+1} \mid y_{m}=0,0<t<T\right\} \subset E_{m+1}$;

- $R_{T}^{+}=\left\{(y, t) \in E_{m+1} \mid y_{m}>0,0<t<T\right\}$;

- $R_{T}^{-}=\left\{(y, t) \in E_{m+1} \mid y_{m}<0,0<t<T\right\}$;

- $E_{m+1}^{+}=\left\{(y, t) \in E_{m+1} \mid y_{m}>0, t>0\right\}$

- $E_{m+1}^{-}=\left\{(y, t) \in E_{m+1} \mid y_{m}<0, t>0\right\}$;

- $R_{t_{1}, t_{2}}^{+}=\left\{(y, t) \in E_{m+1} \mid y_{m}>0,0<t_{1}<t<t_{2} \leq T\right\}$;

- $R_{t_{1}, t_{2}}^{-}=\left\{(y, t) \in E_{m+1} \mid y_{m}<0,0<t_{1}<t<t_{2} \leq T\right\}$.

- $l, n$ : positive integers,

- $0<\lambda<1$.

1.2. Domains. In what follows we suppose that $\Omega_{1}, \Omega$ are domains of Lyapunov type and belong to the class $A^{(n, \lambda)}, n \geq 2$. The latter, for example for the domain $\Omega$, means that there exists a number $r_{1}>0$ such that for every $x \in \partial \Omega$ the set $\partial \Omega \cap \Gamma_{m}\left(x, r_{1}\right)$ can be represented locally in a system of coordinates $\left(y_{1}, \ldots, y_{m}\right)$ with centre at $x$ in the form $y_{m}=$ $\mathcal{Z}\left(y_{1}, \ldots, y_{m-1}\right)$, where $O y_{m}$ coincides with the interior normal to $\partial \Omega$ at $x$ and $\mathcal{Z}$ is a function defined in the projection of $\partial \Omega \cap \Gamma_{m}\left(x, r_{1}\right)$ onto the hyperplane $E_{m-1}$, belongs to the class $C^{(n, \lambda)}$ and vanishes at $x$ together with its first order derivatives. We fix a number $r_{*}$ with

$$
0<r_{*} \leq \min \left(r_{0} / 2, r_{1} / 2\right)
$$

For fixed $x_{*}$ we put

$$
\begin{aligned}
\Omega_{*}^{(q)} & =\Omega^{(q)} \cap \Gamma_{m}\left(x_{*}, r_{*}\right), & \gamma_{*} & =\gamma \cap \Gamma_{m}\left(x_{*}, r_{*}\right), & & x_{*} \in \gamma ; \\
\Omega^{*} & =\Omega \cap \Gamma_{m}\left(x_{*}, r_{*}\right), & \partial_{*} \Omega & =\partial \Omega \cap \Gamma_{m}\left(x_{*}, r_{*}\right), & & x_{*} \in \partial \Omega .
\end{aligned}
$$

For convenience $x_{*}$ is assumed to coincide with the origin, and the $O x_{m}$-axis with the interior normal to $\partial \Omega^{(1)}$ (resp. $\partial \Omega$ ). Then by definition $\gamma_{*}$ can be represented by an equation

$$
x_{m}=\zeta\left(x_{1}, \ldots x_{m-1}\right), \quad \zeta \in C^{(n, \lambda)},
$$

and $\partial_{*} \Omega$ by an equation

$$
x_{m}=\mathcal{Z}\left(x_{1}, \ldots x_{m-1}\right), \quad \mathcal{Z} \in C^{(n, \lambda)} .
$$

A local change of variables

$$
\left\{\begin{array}{l}
y_{s}=x_{s}, \quad s=1, \ldots, m-1 \\
y_{m}=x_{m}-\zeta\left(x_{1}, \ldots x_{m-1}\right)
\end{array}\right.
$$

or

$$
\left\{\begin{array}{l}
y_{s}=x_{s}, \quad s=1, \ldots, m-1 \\
y_{m}=x_{m}-\mathcal{Z}\left(x_{1}, \ldots x_{m-1}\right)
\end{array}\right.
$$


defines a regular transformation which is invertible. Note that the Jacobians of these transformations are equal to 1 . The transformation (1.4) leads to a one-to-one correspondence between $\Omega_{*}^{(q)}$ and a domain $\Delta^{(q)}$ of the space $\left(y_{1}, \ldots, y_{m}\right) \subset E_{m}$, and between $\gamma_{*}$ and a part $\sigma$ of the hyperplane $\left\{x_{m}=0\right\}$ $\subset E_{m}$. Similarly we define the domain $\Delta$ as the image of $\Omega^{*}$ under the transformation (1.5), and the part $\Sigma$ of the hyperplane $\left\{x_{m}=0\right\} \subset E_{m}$ as the image of $\partial_{*} \Omega$. Furthermore, every function of $x$ is transformed by the change of variables (1.4) or (1.5) into a function of $y$ denoted by the same letter with a bar above. We set

$$
j(y)=\left(1+\sum_{s=1}^{m-1} \zeta_{y_{s}}^{2}\right)^{1 / 2}, \quad J(y)=\left(1+\sum_{s=1}^{m-1} \mathcal{Z}_{y_{s}}^{2}\right)^{1 / 2} .
$$

An element of area of the surface $\gamma($ respectively $\partial \Omega$ ) is then given by

$$
d \sigma=j(y) d y_{1} \ldots d y_{m-1}, \quad d \Sigma=J(y) d y_{1} \ldots d y_{m-1} .
$$

We denote by $\gamma_{i}(x)$, respectively $n_{i}(x), i=1, \ldots, m$, the components of the exterior normal to the surface $\gamma$ (respectively $\partial \Omega$ ) with respect to $\Omega^{(1)}$ (respectively $\Omega$ ) at $x$. Then

$$
\gamma_{i}(x)=-\frac{1}{j(y)} \frac{\partial y_{m}}{\partial x_{i}}, \quad n_{i}(x)=-\frac{1}{J(y)} \frac{\partial y_{m}}{\partial x_{i}}, \quad i=1, \ldots, m .
$$

1.3. Function spaces. For a domain $Q \subset E_{m+1}$ and function $u(x, t)$ we define the quantities:

$$
\begin{aligned}
\mathcal{U}_{0}(\bar{Q})= & \max _{(x, t) \in \bar{Q}}|u(x, t)|, \quad \mathcal{U}_{l}(\bar{Q})=\sum_{2 i+j=l} \max _{(x, t) \in \bar{Q}}\left|D_{t}^{i} D_{x}^{j} u(x, t)\right|, \\
\mathcal{U}_{0, \lambda}(\bar{Q})= & \sup _{(x, t),(y, t) \in \bar{Q}} \frac{|u(x, t)-u(y, t)|}{|x-y|^{\lambda}}+\sup _{(x, t),(x, \tau) \in \bar{Q}} \frac{|u(x, t)-u(x, \tau)|}{|t-\tau|^{\lambda / 2}}, \\
\mathcal{U}_{l, \lambda}(\bar{Q})= & \sum_{2 i+j=l} \sup _{(x, t),(y, t) \in \bar{Q}} \frac{\left|D_{t}^{i} D_{x}^{j} u(x, t)-D_{t}^{i} D_{x}^{j} u(y, t)\right|}{|x-y|^{\lambda}} \\
& +\sum_{0<l-2 i-j+\lambda<2} \sup _{(x, t),(x, \tau) \in \bar{Q}} \frac{\left|D_{t}^{i} D_{x}^{j} u(x, t)-D_{t}^{i} D_{x}^{j} u(x, \tau)\right|}{|t-\tau|^{(l-2 i-j+\lambda) / 2}} .
\end{aligned}
$$

We also introduce the function spaces:

- $C^{(n, \lambda)}(\bar{Q}) \equiv C_{x t}^{(n, \lambda)(n / 2, \lambda / 2)}(\bar{Q})$ : the Banach space of functions $u(x, t)$ continuous on $\bar{Q}$ with derivatives $D_{t}^{i} D_{x}^{j} u(2 i+j \leq n)$ uniformly Hölder continuous and having finite norm $\|u\|=\sum_{l=0}^{n} \mathcal{U}_{l}+\mathcal{U}_{n, \lambda}$,

- $C_{0}^{(n, \lambda)}(\bar{Q}) \equiv C_{0 x t}^{(n, \lambda)(n / 2, \lambda / 2)}(\bar{Q})$ : the subspace of functions $u(x, t) \in$ $C^{(n, \lambda)}(\bar{Q})$ that satisfy zero initial data: $\left.\frac{\partial^{j} u}{\partial t^{j}}\right|_{t=0}=0, j=0,1, \ldots,[n / 2]$, 
- $\stackrel{\circ}{C}_{0}^{(n, \lambda)}(\bar{Q})$ : the subspace of functions in $C_{0}^{(n, \lambda)}(\bar{Q})$ with compact support in $Q$, $\bar{Q}_{T}$,

- $u(x, t)=\left(u^{(1)}(x, t), u^{(2)}(x, t)\right)$ : vector-functions defined on the cylinder

$$
\mathcal{U}_{l}=\mathcal{U}_{l}^{(1)}+\mathcal{U}_{l}^{(2)}, \quad \mathcal{U}_{l, \lambda}=\mathcal{U}_{l, \lambda}^{(1)}+\mathcal{U}_{l, \lambda}^{(2)}, \quad 0 \leq l \leq n
$$

where $\mathcal{U}_{l}^{(q)} \equiv \mathcal{U}_{l}\left(\bar{Q}_{T}^{(q)}\right), \mathcal{U}_{l, \lambda}^{(q)} \equiv \mathcal{U}_{l, \lambda}\left(\bar{Q}_{T}^{(q)}\right)$,

- $\mathbf{C}^{(n, \lambda)}\left(\bar{Q}_{T}\right) \equiv C^{(n, \lambda)}\left(\bar{Q}_{T}^{(1)}\right) \dot{+} C^{(n, \lambda)}\left(\bar{Q}_{T}^{(2)}\right)$ : the direct sum space of vector-functions $u(x, t)=\left(u^{(1)}(x, t), u^{(2)}(x, t)\right)$ with $u^{(q)} \in C^{(n, \lambda)}\left(\bar{Q}_{T}^{(q)}\right)$, having the finite norm

$$
\|u\|=\sum_{l=0}^{n} \mathcal{U}_{l}+\mathcal{U}_{n, \lambda} .
$$

- Let $\Omega \in A^{(n, \lambda)}$ and let $\varphi(x, t)$ be a function defined on the lateral surface $\partial_{T} \Omega$ of the cylinder $Q_{T}$. We define the space $C^{(n, \lambda)}\left(\partial_{T} \Omega\right) \equiv$ $C_{x t}^{(n, \lambda)(n / 2, \lambda / 2)}\left(\partial_{T} \Omega\right)$ as the set of functions $\varphi(x, t)$ with finite norm $\Phi_{0}\left(\partial_{T} \Omega\right)$ $+\Phi_{n, \lambda}\left(\partial_{T} \Omega\right)$, where $\Phi_{n, \lambda}$ has the analogous meaning to $\mathcal{U}_{n, \lambda}$. It is known that $\varphi(x, t)$ can be extended to $Q_{T}$ (if $n \geq 1$ ) so that the extended function belongs to $C^{(n, \lambda)}\left(Q_{T} \cup \partial_{T} \Omega\right)$; moreover, by means of the same construction this extension can be made for all functions $\varphi \in C^{(n, \lambda)}\left(\partial_{T} \Omega\right)$ so that the norms $\Phi_{0}\left(Q_{T} \cup \partial_{T} \Omega\right)+\Phi_{n, \lambda}\left(Q_{T} \cup \partial_{T} \Omega\right)$ and $\Phi_{0}\left(\partial_{T} \Omega\right)+\Phi_{n, \lambda}\left(\partial_{T} \Omega\right)$ are equivalent. In view of this, saying that $\varphi \in C^{(n, \lambda)}\left(\partial_{T} \Omega\right), n \geq 1$, implies that $\varphi \in C^{(n, \lambda)}\left(Q_{T} \cup \partial_{T} \Omega\right)$.

- If $Q_{T}^{(1)} \in A^{(n, \lambda)}$, the function $\chi(x, t)$ is given on the surface $\gamma_{T}$ and $\chi(x, t) \in C^{(n, \lambda)}\left(\gamma_{T}\right)$, this will mean that there exist functions $\chi^{(q)}(x, t) \in$ $C^{(n, \lambda)}\left(Q_{T}^{(q)} \cup \gamma_{T}\right), q=1,2$, such that $\chi(x, t)=\left.\chi^{(1)}(x, t)\right|_{\gamma_{T}}-\left.\chi^{(2)}(x, t)\right|_{\gamma_{T}}$, where

$$
\left.\chi^{(q)}(x, t)\right|_{\gamma_{T}}=\lim _{Q_{T}^{(q)} \ni(y, \tau) \rightarrow(x, t) \in \gamma_{t}} \chi^{(q)}(y, \tau), \quad q=1,2 .
$$

For $f=\left(f^{(1)}, f^{(2)}\right)$ the symbol $[f]_{\gamma_{T}}=\left.f^{(1)}\right|_{\gamma_{T}}-\left.f^{(2)}\right|_{\gamma_{T}}$ will denote the jump of the function $f$ when crossing $\gamma_{T}$.

- If a vector-function $\omega(x)$ is defined on $\bar{\Omega}$, then we shall consider it in the space $C^{(n, \lambda)}(\bar{\Omega})$ with the norm $\Omega_{0}+\Omega_{n, \lambda}$.

We now state some inequality for the above introduced quantities.

Lemma 1.1 (see [6, Chapt. II, Lemma 3.2]). Suppose that the domain $\Omega$ satisfies the cone condition, that is, for every point $\xi \in \partial \Omega$ there exists a finite right circular cone $K$ with vertex $\xi$ and some altitude $d>0$ such that 
$\bar{K} \cap \bar{\Omega}=\xi$. Then for any $u(x, t) \in C^{(n, \lambda)}\left(\bar{Q}_{T}\right)$ we have

$$
\begin{aligned}
\mathcal{U}_{l} & =\mathcal{O}\left(\delta^{n-l+\lambda} \mathcal{U}_{n, \lambda}+\delta^{-l} \mathcal{U}_{0}\right), \\
\mathcal{U}_{l, \lambda} & =\mathcal{O}\left(\delta^{n-l} \mathcal{U}_{n, \lambda}+\delta^{-(l+\lambda)} \mathcal{U}_{0}\right), \quad 0 \leq l \leq n,
\end{aligned}
$$

for any $\delta \in(0, \min (d, \sqrt{T}))$, where the estimating constants depend only on $l, n, \lambda, m$ and on the solid angle of the cone $K$.

Lemma 1.2 (cf. [7, (33)] and [4, estimates (5)-(10)]). Let $\Omega \in A^{(n, \lambda)}$. For all functions $u(x, t) \in C^{(2, \lambda)}\left(\bar{Q}_{T}\right)$ the following inequalities hold uniformly:

$$
\begin{aligned}
\mathcal{U}_{l} & =\mathcal{O}\left(\mathcal{U}_{2, \lambda}^{\frac{1}{2+\lambda}} \mathcal{U}_{0}^{\frac{2-l+\lambda}{2+\lambda}}+\mathcal{U}_{0}\right), & & 0<l \leq 2, \\
\mathcal{U}_{l, \lambda} & =\mathcal{O}\left(\mathcal{U}_{2, \lambda}^{\frac{l+\lambda}{2+\lambda}} \mathcal{U}_{0}^{\frac{2-l}{2+\lambda}}+\mathcal{U}_{0}\right), & & 0<l \leq 2,
\end{aligned}
$$

where the estimating constants depend only on $\Omega$ and $T$.

Proof. If $l=1$, then (1.11) follows from [7, (33.8)]. Let $l=2$. In view of [7, (33.3)], we have

$$
\begin{aligned}
\max _{t \in[0, T]}\left|\frac{\partial u}{\partial t}\right|= & \mathcal{O}\left\{\left[\sup _{t, t^{\prime} \in[0, T]} \frac{\left|D_{t} u(x, t)-D_{t} u\left(x, t^{\prime}\right)\right|}{\left|t-t^{\prime}\right|^{\alpha}}\right]^{\frac{1}{1+\alpha}}\left[\max _{t \in[0, T]}|u(x, t)|\right]^{\frac{\alpha}{1+\alpha}}\right. \\
& \left.+\frac{1}{T} \max _{t \in[0, T]}|u(x, t)|\right\}, \quad \forall x \in \Omega, \alpha=\frac{\lambda}{2},
\end{aligned}
$$

whence it follows that

$$
\max _{(x, t) \in \bar{Q}_{T}}\left|\frac{\partial u}{\partial t}\right|=\mathcal{O}\left(\mathcal{U}_{2, \lambda}^{\frac{2}{2+\lambda}} \mathcal{U}_{0}^{\frac{\lambda}{2+\lambda}}+\mathcal{U}_{0}\right) .
$$

This inequality together with $[7,(33.8)]$ gives (1.11).

We now prove inequality (1.12). Let $l=1$ and let $D_{x} u$ denote the partial derivative of $u(x, t)$ with respect to any space variable. Then

$$
\begin{aligned}
& \frac{\left|D_{x} u(x, t)-D_{x} u\left(x, t^{\prime}\right)\right|}{\left|t-t^{\prime}\right|^{\frac{\lambda}{2}}} \\
& \quad=\left[\frac{\left|D_{x} u(x, t)-D_{x} u\left(x, t^{\prime}\right)\right|}{\left|t-t^{\prime}\right|^{\frac{1+\lambda}{2}}}\right]^{\frac{\lambda}{1+\lambda}}\left|D_{x} u(x, t)-D_{x} u\left(x, t^{\prime}\right)\right|^{\frac{1}{1+\lambda}},
\end{aligned}
$$

whence

$$
\sup _{(x, t),\left(x, t^{\prime}\right) \in Q_{T}} \frac{\left|D_{x} u(x, t)-D_{x} u\left(x, t^{\prime}\right)\right|}{\left|t-t^{\prime}\right|^{\frac{\lambda}{2}}}=\mathcal{O}\left(\mathcal{U}_{2, \lambda}^{\frac{\lambda}{1+\lambda}} \mathcal{U}_{1}^{\frac{1}{1+\lambda}}\right) .
$$

Applying Young's inequality with $p=\frac{(1+\lambda)^{2}}{\lambda(2+\lambda)}, p^{\prime}=(1+\lambda)^{2}$, we deduce from (1.13) and (1.11) with $l=1$ that

$$
\sup _{(x, t),\left(x, t^{\prime}\right) \in Q_{T}} \frac{\left|D_{x} u(x, t)-D_{x} u\left(x, t^{\prime}\right)\right|}{\left|t-t^{\prime}\right|^{\frac{\lambda}{2}}}=\mathcal{O}\left(\mathcal{U}_{2, \lambda}^{\frac{1+\lambda}{2+\lambda}} \mathcal{U}_{0}^{\frac{1}{2+\lambda}}+\mathcal{U}_{0}\right) .
$$


Let us now fix $\delta \in(0, \sqrt{T}]$. For any $x \in \Omega$ the following is trivial:

$$
\begin{aligned}
& \frac{\left|u(x, t)-u\left(x, t^{\prime}\right)\right|}{\left|t-t^{\prime}\right|^{\frac{1+\lambda}{2}}} \\
& \quad \leq \begin{cases}\left|D_{t} u(x, \tau)\right|_{t<\tau<t^{\prime}}|\cdot| t-\left.t^{\prime}\right|^{\frac{1-\lambda}{2}} \leq \delta^{1-\lambda} \mathcal{U}_{2}, & \left|t-t^{\prime}\right| \leq \delta^{2}, \\
\delta^{-(1+\lambda)} \operatorname{osc}_{t} u(x, t), & \left|t-t^{\prime}\right| \geq \delta^{2} .\end{cases}
\end{aligned}
$$

Hence

$$
\sup _{(x, t),\left(x, t^{\prime}\right) \in Q_{T}} \frac{\left|u(x, t)-u\left(x, t^{\prime}\right)\right|}{\left|t-t^{\prime}\right|^{\frac{1+\lambda}{2}}} \leq \min _{\delta \in(0, \sqrt{T}]} \theta(\delta),
$$

where $\theta(\delta)=\max \left(\delta^{1-\lambda} \mathcal{U}_{2}, \delta^{-(1+\lambda)} \sup _{x \in \Omega} \operatorname{osc}_{t} u(x, t)\right)$. By the trivial inequalities

$$
\sup _{x \in \Omega} \operatorname{osc}_{t} u(x, t) \leq T \mathcal{U}_{2}, \quad \sup _{x \in \Omega} \operatorname{osc}_{t} u(x, t) \leq 2 \mathcal{U}_{0}
$$

we have

$$
\begin{aligned}
\delta^{-(1+\lambda)} \sup _{x \in \Omega} \operatorname{osc}_{t} u(x, t) & =\delta^{-(1+\lambda)}\left(\sup _{x \in \Omega} \operatorname{osc}_{t} u(x, t)\right)^{\frac{1+\lambda}{2}}\left(\sup _{x \in \Omega} \operatorname{osc}_{t} u(x, t)\right)^{\frac{1-\lambda}{2}} \\
& \leq\left(\delta^{-1} \sqrt{T}\right)^{1+\lambda} \mathcal{U}_{2}^{\frac{1+\lambda}{2}}\left(2 \mathcal{U}_{0}\right)^{\frac{1-\lambda}{2}}
\end{aligned}
$$

and hence, $\theta(\delta)$ achieves a minimum at $\delta=\sqrt{T}$, i.e.

$$
\min _{\delta \in(0, \sqrt{T}]} \theta(\delta)=\mathcal{U}_{2}^{\frac{1+\lambda}{2}}\left(2 \mathcal{U}_{0}\right)^{\frac{1-\lambda}{2}} .
$$

Consequently, by (1.15) we have

$$
\sup _{(x, t),\left(x, t^{\prime}\right) \in Q_{T}} \frac{\left|u(x, t)-u\left(x, t^{\prime}\right)\right|}{\left|t-t^{\prime}\right|^{\frac{1+\lambda}{2}}}=\mathcal{O}\left(\mathcal{U}_{2}^{\frac{1+\lambda}{2}} \mathcal{U}_{0}^{\frac{1-\lambda}{2}}\right) .
$$

From (1.16) and (1.11) for $l=2$ we obtain

$$
\sup _{(x, t),\left(x, t^{\prime}\right) \in Q_{T}} \frac{\left|u(x, t)-u\left(x, t^{\prime}\right)\right|}{\left|t-t^{\prime}\right|^{\frac{1+\lambda}{2}}}=\mathcal{O}\left\{\mathcal{U}_{2, \lambda}^{\frac{1+\lambda}{2+\lambda}} \mathcal{U}_{0}^{\frac{1}{2+\lambda}}+\mathcal{U}_{0}\right\} .
$$

Inequalities (1.14), (1.17) together with [7, (33.9)] imply (1.12) if $l=1$. Finally, if $l=0$ we have

$$
\frac{\left|u(x, t)-u\left(x, t^{\prime}\right)\right|}{\left|t-t^{\prime}\right|^{\frac{\lambda}{2}}}=\left(\frac{\left|u(x, t)-u\left(x, t^{\prime}\right)\right|}{\left|t-t^{\prime}\right|^{\frac{1+\lambda}{2}}}\right)^{\frac{\lambda}{1+\lambda}}\left|u(x, t)-u\left(x, t^{\prime}\right)\right|^{\frac{1}{1+\lambda}},
$$

therefore by (1.12) for $l=1$ we get

$$
\begin{aligned}
\sup _{(x, t),\left(x, t^{\prime}\right) \in Q_{T}} \frac{\left|u(x, t)-u\left(x, t^{\prime}\right)\right|}{\left|t-t^{\prime}\right|^{\frac{\lambda}{2}}} & =\mathcal{O}\left(\mathcal{U}_{1, \lambda}^{\frac{\lambda}{1+\lambda}} \mathcal{U}_{0}^{\frac{1}{1+\lambda}}\right) \\
& =\mathcal{O}\left(\mathcal{U}_{2, \lambda}^{\frac{\lambda}{2+\lambda}} \mathcal{U}_{0}^{\frac{2}{2+\lambda}}+\mathcal{U}_{0}\right) .
\end{aligned}
$$

This together with $[7,(33.9)]$ proves $(1.12)$ for $l=0$. Lemma 1.2 is proved. 
1.4. Local cylinders. We define local neighbourhoods of the surface $\gamma_{T}$. For $0<\tau<T, 0<t_{1}<t_{2}<T$ we put:

- $Q_{* \tau}^{(q)}=\Omega_{*}^{(q)} \times(0, \tau], \quad Q_{* t_{1}, t_{2}}^{(q)}=\Omega_{*}^{(q)} \times\left(t_{1}, t_{2}\right), \quad q=1,2$;

- $\gamma_{* \tau}=\gamma_{*} \times(0, \tau], \quad \gamma_{* t_{1}, t_{2}}=\gamma_{*} \times\left(t_{1}, t_{2}\right)$;

- $Q_{* \tau}=Q_{* \tau}^{(1)} \cup Q_{* \tau}^{(2)} \cup \gamma_{* \tau}, \quad Q_{* t_{1}, t_{2}}=Q_{* t_{1}, t_{2}}^{(1)} \cup Q_{* t_{1}, t_{2}}^{(2)} \cup \gamma_{* t_{1}, t_{2}}$

(here the domains with a star are defined by (1.3)). By means of the local transformation (1.4) we find the images of these sets:

$$
\begin{aligned}
p_{\tau}^{(q)} & =\Delta^{(q)} \times(0, \tau], & & p_{t_{1}, t_{2}}^{(q)}=\Delta^{(q)} \times\left(t_{1}, t_{2}\right), \\
\sigma_{\tau} & =\sigma \times(0, \tau], & & \sigma_{t_{1}, t_{2}}=\sigma \times\left(t_{1}, t_{2}\right) .
\end{aligned}
$$

We consider domains that are symmetric with respect to the $(m-1)$ dimensional hyperplane $\left\{y_{m}=0\right\} \subset E_{m}$ :

$$
\widetilde{\Delta}^{(q)} \subseteq \Delta^{(q)}, \quad q=1,2, \quad \partial \widetilde{\Delta}^{(1)} \cap \partial \widetilde{\Delta}^{(2)}=\sigma
$$

and the corresponding sets

$$
\begin{aligned}
\widetilde{p}_{\tau}^{(q)} & =\widetilde{\Delta}^{(q)} \times(0, \tau], \quad \widetilde{p}_{t_{1}, t_{2}}^{(q)}=\widetilde{\Delta}^{(q)} \times\left(t_{1}, t_{2}\right), \quad q=1,2, \\
\widetilde{p}_{\tau} & =\widetilde{p}_{\tau}^{(1)} \cup \widetilde{p}_{\tau}^{(2)} \cup \sigma_{\tau}, \quad \tau \in(0, T), \\
\widetilde{p}_{t_{1}, t_{2}} & =\widetilde{p}_{t_{1}, t_{2}}^{(1)} \cup \widetilde{p}_{t_{1}, t_{2}}^{(2)} \cup \sigma_{t_{1}, t_{2}}, \quad 0<t_{1}<t_{2}<T .
\end{aligned}
$$

We also consider an infinite set of pairs of points $P_{0}=\left(P_{0}^{(1)}, P_{0}^{(2)}\right)$ with $P_{0}^{(q)}=\left(y_{0}^{(q)}\right) \in \widetilde{\Delta}^{(q)}$ which are symmetric with respect to $\sigma$ and where $P_{0}^{(q)} \in \widetilde{\Delta}^{(q)}, q=1,2$, can be set in correspondence with each point $P_{0}^{\prime}=$ $\left(y_{0}^{\prime}, 0,0\right) \in \sigma$. We put

$$
\mathcal{N}=\left(\partial \widetilde{\Delta}^{(1)} \cap \partial \widetilde{\Delta}^{(2)}\right) \backslash \sigma, \quad \mathcal{N}_{\tau}=\mathcal{N} \times(0, \tau], \quad \mathcal{N}_{t_{1}, t_{2}}=\mathcal{N} \times\left(t_{1}, t_{2}\right) .
$$

For each $P_{0}=\left(P_{0}^{(1)}, P_{0}^{(2)}\right)$ with $P_{0}^{(q)} \in \widetilde{\Delta}^{(q)} \backslash \mathcal{N}(q=1,2)$, we put

$$
r\left(P_{0}, \mathcal{N}\right)=4 d\left(P_{0}\right), \quad \varkappa d\left(P_{0}\right)=\varrho, \forall \varkappa \leq \varkappa_{0}<1
$$

where $r\left(P_{0}, \mathcal{N}\right)=\inf _{P \in \mathcal{N}} P_{0} P$ denotes the distance from $P_{0}=\left(y_{0}, 0\right)$ to the set $\mathcal{N}$, and by the symmetry of $\widetilde{\Delta}^{(1)}$ and $\widetilde{\Delta}^{(2)}$ we have $r\left(P_{0}, \mathcal{N}\right)=$ $r\left(P_{0}^{(1)}, \mathcal{N}\right)=r\left(P_{0}^{(2)}, \mathcal{N}\right)$. We fix a number $k>3$ and denote by $\Gamma_{m}\left(P_{0}, k \varrho\right)$ the pair of $m$-dimensional hyperspheres

$$
\Gamma_{m}\left(P_{0}, k \varrho\right)=\left\{\Gamma_{m}\left(P_{0}^{(1)}, k \varrho\right), \Gamma_{m}\left(P_{0}^{(2)}, k \varrho\right)\right\} .
$$

It is easy to see that $\Gamma_{m}\left(P_{0}^{(1)}, k \varrho\right) \cap \sigma=\Gamma_{m}\left(P_{0}^{(2)}, k \varrho\right) \cap \sigma$. If $k \varkappa<4$ then by $(1.23), \Gamma_{m}\left(P_{0}, k \varrho\right)$ has points in common with $\partial \widetilde{\Delta}^{(1)} \cup \partial \widetilde{\Delta}^{(2)}$ only on $\sigma$. The set of these points forms a hypersphere of dimension $m-1$ with centre at $P_{0}^{\prime}$. Its radius is denoted by $\nu d\left(P_{0}^{\prime}\right)$. 
Now we define

$$
\begin{aligned}
c_{\tau}^{(q)(\beta)} & =\overline{\widetilde{p}_{\tau}^{(q)}} \cap\left\{\Gamma_{m}\left(P_{0}, \beta d\left(P_{0}\right)\right) \times[0, \tau]\right\}, \\
c_{t_{1}, t_{2}}^{(q)(\beta)} & =\overline{\widetilde{p}_{t_{1}, t_{2}}^{(q)}} \cap\left\{\Gamma_{m}\left(P_{0}, \beta d\left(P_{0}\right)\right) \times\left[t_{1}, t_{2}\right]\right\}, \\
\sigma_{\tau}^{(\beta)} & =\left\{\sigma \cap \Gamma_{m}\left(P_{0}, \beta d\left(P_{0}\right)\right)\right\} \cap[0, \tau], \\
\sigma_{t_{1}, t_{2}}^{(\beta)} & =\left\{\sigma \cap \Gamma_{m}\left(P_{0}, \beta d\left(P_{0}\right)\right)\right\} \cap\left[t_{1}, t_{2}\right], \quad \forall \beta, 0<\beta<4 .
\end{aligned}
$$

Later on we shall distinguish three kind of cylinders in the neighbourhood of the surface $\sigma_{T}$ :

(1) the cylinders $c_{\tau}^{(\varkappa)}, c_{\tau}^{(k \varkappa)}$ that adjoin the surfaces $\widetilde{\Delta}^{(q)}, \sigma_{\tau}$; these cylinders are sets from (1.24) if $\varrho>r\left(P_{0}, \sigma\right)$;

(2) the cylinders $c_{t_{1}, t_{2}}^{(\varkappa)}, c_{t_{1}, t_{2}}^{(k \varkappa)}$ that adjoin only the surface $\sigma_{t_{1}, t_{2}}$ and do not have common points with the hyperplane $\{t=0\}$; they are obtained from (1.24) if $\varrho>r\left(P_{0}, \sigma\right), t_{2}>t_{1}>0$;

(3) the cylinders $c_{\tau}^{(\varkappa)}, c_{\tau}^{(k \varkappa)}$ that adjoin the lower base of the cylinder $Q_{T}$, i.e. the set $\{t=0\}$, and do not have common points with $\sigma_{\tau}$; they are sets from (1.24) if $k \varrho<r\left(P_{0}, \sigma\right)$.

Similarly, we consider local cylinders of three kinds in the neighbourhood of the surface $\partial_{T} \Omega$.

If $v(y, t) \in \mathbf{C}^{(n, \lambda)}\left(\bar{Q}_{T}\right)$, then for any point $P_{0}=\left(P_{0}^{(1)}, P_{0}^{(2)}\right)$ with $P_{0}^{(q)} \in$ $\overline{\widetilde{p}_{\tau}^{(q)}} \backslash \mathcal{N}_{\tau}, q=1,2$, and any number $\beta \in(0,4)$ and integer $l \in[0, n]$ we define

$$
\begin{aligned}
V_{(\tau) l}^{(q)(\beta)} & =V_{l}^{(q)}\left(c_{\tau}^{(q)(\beta)}\right), & & V_{(\tau) l, \lambda}^{(q)(\beta)}=V_{l, \lambda}^{(q)(\beta)}\left(c_{\tau}^{(q)(\beta)}\right), \\
\mathcal{V}_{(\tau) l}^{(\beta)} & =V_{(\tau) l}^{(1)(\beta)}+V_{(\tau) l}^{(2)(\beta)}, & \mathcal{V}_{(\tau) l, \lambda}^{(\beta)} & =V_{(\tau) l, \lambda}^{(1)(\beta)}+V_{(\tau) l, \lambda}^{(2)(\beta)} .
\end{aligned}
$$

If $\varphi(y, t) \in C^{(n, \lambda)}(\Pi)$, then for any point $P_{0}^{\prime} \in \sigma$ we set

$$
\Phi_{(\tau) l}^{(\beta)}\left(P_{0}^{\prime}\right)=\Phi_{l}\left(\sigma_{\tau}^{(\beta)}\left(P_{0}^{\prime}\right)\right), \quad \Phi_{(\tau) l, \lambda}^{(\beta)}\left(P_{0}^{\prime}\right)=\Phi_{l, \lambda}\left(\sigma_{\tau}^{(\beta)}\left(P_{0}^{\prime}\right)\right) .
$$

Similarly, we define the quantities $\mathcal{V}_{\left(t_{1}, t_{2}\right) l}^{(\beta)}, \mathcal{V}_{\left(t_{1}, t_{2}\right) l, \lambda}^{(\beta)}$. If $\omega(x) \in \mathbf{C}^{(n, \lambda)}(\bar{\Omega})$, then for any point $P_{0} \in \overline{\widetilde{\Delta}}$ we put:

$$
\begin{aligned}
\Omega_{l}^{(q)(\beta)} & =\Omega_{l}^{(q)}\left\{\overline{\widetilde{\Delta}^{(q)}} \cap \Gamma_{m}\left(P_{0}, \beta d\left(P_{0}\right)\right)\right\}, \\
\Omega_{l, \lambda}^{(q)(\beta)} & =\Omega_{l, \lambda}^{(q)}\left\{\overline{\widetilde{\Delta}^{(q)}} \cap \Gamma_{m}\left(P_{0}, \beta d\left(P_{0}\right)\right)\right\}, \\
\Omega_{l}^{(\beta)} & =\Omega_{l}^{(1)(\beta)}+\Omega_{l}^{(2)(\beta)}, \quad \Omega_{l, \lambda}^{(\beta)}=\Omega_{l, \lambda}^{(1)(\beta)}+\Omega_{l, \lambda}^{(2)(\beta)} .
\end{aligned}
$$

Later on we shall choose the number $\varkappa_{0}<1$ from (1.23) so that $k \varkappa<4$, $\nu \leq 3$. 
Finally, we define the following quantities provided that $\tau=t_{2}-t_{1}=\varrho^{2}$ :

$$
\begin{aligned}
d_{0} & =\sup d\left(P_{0}\right), & & \\
B_{2}^{(q)(\varkappa)} & =\sup \varrho^{2} V_{2}^{(q)(\varkappa)}, & B_{2, \lambda}^{(q)(\varkappa)} & =\sup \varrho^{2+\lambda} V_{2, \lambda}^{(q)(\varkappa)}, \\
\mathcal{B}_{2}^{(\varkappa)} & =B_{2}^{(1)(\varkappa)}+B_{2}^{(2)(\varkappa)}, & \mathcal{B}_{2, \lambda}^{(\varkappa)} & =B_{2, \lambda}^{(1)(\varkappa)}+B_{2, \lambda}^{(2)(\varkappa)}
\end{aligned}
$$

(here the sup is taken over all points $P_{0}=\left(P_{0}^{(1)}, P_{0}^{(2)}\right)$ with $P_{0}^{(q)} \in \widetilde{\Delta}^{(q)} \backslash \mathcal{N}$, $q=1,2)$. For the above-defined quantities the following assertions are valid.

Lemma 1.3 (cf. [7, Chapt. VIII, §33]). If $k \varkappa<4$ then

$$
\mathcal{B}_{2, \lambda}^{(k \varkappa)}=\mathcal{O}\left\{k^{2+\lambda}\left(\mathcal{B}_{2, \lambda}^{(\varkappa)}+\mathcal{B}_{2}^{(\varkappa)}\right)\right\}
$$

uniformly with respect to $\varkappa$.

Proof. We prove (1.29) e.g. for the quantities that are defined on the cylinders $c_{\tau}^{(k \varkappa)}$. Covering the base of the cylinder $c_{\tau}^{(q)(k \varkappa)}$ by a finite number $n_{q}$ of balls $\Gamma_{m}$ of radius $\varrho=\varkappa d\left(P_{0}\right)$ we have

$$
\begin{aligned}
& \sup _{P_{0}^{(q)} \in \widetilde{\Delta}^{(q)} \backslash \mathcal{N}}(k \varrho)^{2+\lambda} \sup _{(y, t),(\bar{y}, t) \in c_{\tau}^{(q)(k \varkappa)}\left(P_{0}^{(q)}\right)} \frac{\left|D_{y}^{2} v^{(q)}(y, t)-D_{y}^{2} v^{(q)}(\bar{y}, t)\right|}{|y-\bar{y}|^{\lambda}} \\
& \leq \sup _{P_{0}^{(q)} \in \widetilde{\Delta}^{(q)} \backslash \mathcal{N}}(k \varrho)^{2+\lambda} \sup _{\substack{\forall y, \bar{y}:|y-\bar{y}| \leq \varrho, t \in[0, \tau]}} \frac{\left|D_{y}^{2} v^{(q)}(y, t)-D_{y}^{2} v^{(q)}(\bar{y}, t)\right|}{|y-\bar{y}|^{\lambda}} \\
& +\sup _{P_{0}^{(q)} \in \widetilde{\Delta}^{(q)} \backslash \mathcal{N}}(k \varrho)^{2+\lambda} \sup _{\substack{\forall y, \bar{y}:|y-\bar{y}|>\varrho,(y, t),(\bar{y}, t) \in c_{\tau}^{(q)(k \varkappa)}\left(P_{0}^{(q)}\right)}} \frac{\left|D_{y}^{2} v^{(q)}(y, t)-D_{y}^{2} v^{(q)}(\bar{y}, t)\right|}{|y-\bar{y}|^{\lambda}} \\
& \leq k^{2+\lambda} B_{2, \lambda}^{(q)(\varkappa)}+k^{2+\lambda} \sup _{P_{0}^{(q)} \in \widetilde{\Delta}^{(q)} \backslash \mathcal{N}} \varrho^{2} 2 \sup _{(y, t) \in c_{\tau}^{(q)(k \varkappa)}\left(P_{0}^{(q)}\right)}\left|D_{y}^{2} v^{(q)}(y, t)\right| \\
& =\mathcal{O}\left\{k^{2+\lambda}\left(B_{2, \lambda}^{(q)(\varkappa)}+2 B_{2}^{(q)(\varkappa)}\right)\right\}, \quad q=1,2,
\end{aligned}
$$

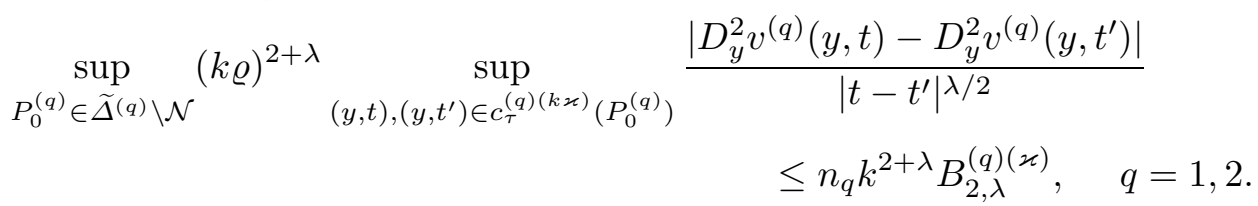

Similarly, we estimate the remaining quantities that define $\mathcal{B}_{2, \lambda}^{(q)(\varkappa)}$. Summing the resulting inequalities over $q=1,2$, we get (1.29).

Lemma 1.4 (cf. [7, Chapt. VIII, §33]). For any closed domain $\pi_{\tau} \subset \widetilde{p}_{\tau} \backslash$ $\mathcal{N}_{\tau}$ such that $\operatorname{dist}\left(\widetilde{p}_{\tau}, \mathcal{N}_{\tau}\right)=\delta>0$, if $\varkappa<4$, then

$$
\mathcal{V}_{2, \lambda}\left(\pi_{\tau}\right)=\mathcal{O}\left\{(\varkappa \delta)^{-(2+\lambda)}\left(\mathcal{B}_{2, \lambda}^{(\varkappa)}+2 \mathcal{B}_{2}^{(\varkappa)}\right)\right\} \text {. }
$$


Proof. We cover the base of the cylinder $\pi_{\tau}^{(q)}$ with a finite number $n_{q}$ of balls $\Gamma_{m}$ of radius $\varrho=\varkappa d\left(P_{0}^{(q)}\right)$ with centre at $P_{0}^{(q)} \in \pi_{\tau}^{(q)} \cap\{t=0\}$. Then by analogy to Lemma 1.3 we obtain

$$
\left.B_{2, \lambda}\left(\pi_{\tau}^{(q)}\right)=\mathcal{O}\left\{B_{2, \lambda}^{(q)(\varkappa)}+2 B_{2}^{(q)(\varkappa)}\right)\right\}, \quad q=1,2 .
$$

On the other hand, by definition we have

$$
B_{2, \lambda}\left(\pi_{\tau}^{(q)}\right)=\sup _{P_{0}^{(q)} \in \pi_{\tau}^{(q)}}\left(\varkappa d\left(P_{0}^{(q)}\right)\right)^{2+\lambda} V_{2, \lambda}\left(\pi_{\tau}^{(q)}\right) .
$$

But by $(1.23)$ we have $d\left(P_{0}^{(q)}\right)=\frac{1}{4} r\left(P_{0}^{(q)}, \mathcal{N}\right)$, and by the assumptions of the lemma $r\left(\pi_{\tau}, \mathcal{N}_{\tau}\right)=\delta>0$, whence $r\left(P_{0}^{(q)}, \mathcal{N}\right) \geq \delta$ for all $P_{0}^{(q)} \in \pi_{\tau}^{(q)}$ and therefore $\varkappa d\left(P_{0}^{(q)}\right)=\frac{\varkappa}{4} r\left(P_{0}^{(q)}, \mathcal{N}\right) \geq \frac{\varkappa}{4} \delta$. Then from (1.31), (1.32) it follows that

$$
\left.\left(\frac{\varkappa}{4} \delta\right)^{2+\lambda} V_{2, \lambda}\left(\pi_{\tau}^{(q)}\right)=\mathcal{O}\left\{B_{2, \lambda}^{(q)(\varkappa)}+2 B_{2}^{(q)(\varkappa)}\right)\right\}, \quad q=1,2 .
$$

The inequality (1.30) follows.

1.5. Formulation of the problems. Assumptions. The main result. We consider the following boundary value problem:

(I)

$$
\left\{\begin{array}{l}
\mathcal{L}^{(q)}\left(x, t, D_{x}\right) u^{(q)} \equiv \frac{\partial u^{(q)}}{\partial t}+\sum_{i, j=1}^{m} a_{i, j}^{(q)}(x, t) u_{x_{i} x_{j}}^{(q)}+\sum_{i=1}^{m} b_{i}^{(q)}(x, t) u_{x_{i}}^{(q)} \\
\quad+c^{(q)}(x, t) u^{(q)}=f^{(q)}(x, t), \quad(x, t) \in Q_{T}^{(q)}, q=1,2 ; \\
{[u]_{\gamma_{T}}=\chi(x, t) ;} \\
{\left[\sum_{i=1}^{m} \tau_{i}(x, t) u_{x_{i}}+\sigma(x, t) u\right]_{\gamma_{T}}=\psi(x, t), \quad(x, t) \in \gamma_{T} ;} \\
\sum_{i=1}^{m} \nu_{i}(x, t) u_{x_{i}}^{(2)}+\left.\mu(x, t) u^{(2)}\right|_{\partial_{T} \Omega}=\varphi(x, t), \quad(x, t) \in \partial_{T} \Omega ; \\
u^{(q)}(x, 0)=\omega^{(q)}(x), \quad x \in \overline{\Omega^{(q)}}, q=1,2 .
\end{array}\right.
$$

We shall need the following

Assumptions I. (1) Uniform parabolicity:

$$
\nu^{(q)} \sum_{i=1}^{m} \xi_{i}^{2} \leq \sum_{i, j=1}^{m} a_{i, j}^{(q)}(x, t) \xi_{i} \xi_{j} \leq \mu^{(q)} \sum_{i=1}^{m} \xi_{i}^{2}, \quad(x, t) \in \overline{Q_{T}^{(q)}}, \quad q=1,2,
$$

where $\nu^{(q)}, \mu^{(q)}$ are positive constants. 
(2) Smoothness:

$$
\mathcal{A}_{0}+\mathcal{T}_{0}+\mathcal{N}_{0}+\Sigma_{0}+\mathcal{M}_{0}=\mathcal{O}(1)
$$

where $\mathcal{A}_{0}, \mathcal{T}_{0}, \mathcal{N}_{0}, \Sigma_{0}, \mathcal{M}_{0}$ relate to $a_{i, j}(x, t), \tau_{i}(x, t), \nu_{i}(x, t), \sigma(x, t), \mu(x, t)$ and are defined with regard to (1.9);

$$
\begin{gathered}
a_{i, j}(x, t), b_{i}(x, t), c(x, t), f(x, t) \in \mathbf{C}^{(0, \lambda)}\left(\bar{Q}_{T}\right), \\
\tau_{i}(x, t), \sigma(x, t) \in \mathbf{C}^{(1, \lambda)}\left(\bar{Q}_{T}\right), \quad \psi(x, t) \in C^{(1, \lambda)}\left(\gamma_{T}\right), \\
\nu_{i}(x, t), \mu(x, t), \varphi(x, t) \in C^{(1, \lambda)}\left(\partial_{T} \Omega\right), \\
\chi(x, t) \in C^{(2, \lambda)}\left(\gamma_{T}\right), \quad \omega(x) \in \mathbf{C}^{(2, \lambda)}(\bar{\Omega}) .
\end{gathered}
$$

(3) Complementarity (see [8]):

$$
\begin{gathered}
\sum_{i=1}^{m} \tau_{i}^{(q)}(x, t) \gamma_{i}(x) \geq \nu_{0}^{(q)}=\mathrm{const}>0, \quad(x, t) \in \gamma_{T}, q=1,2, \\
\sum_{i=1}^{m} \nu_{i}(x, t) n_{i}(x) \geq \nu_{0}=\mathrm{const}>0, \quad(x, t) \in \partial_{T} \Omega .
\end{gathered}
$$

(4) Compatibility (zero-order compatibility conditions for initial and boundary data, see [8]):

$$
\begin{gathered}
{\left[\sum_{i, j=1}^{m} a_{i, j}(x, 0) \omega_{x_{i} x_{j}}(x)+\sum_{i=1}^{m} b_{i}(x, 0) \omega_{x_{i}}(x)+c(x, 0) \omega(x)-f(x, 0)\right]_{\gamma}} \\
=\frac{\partial \chi(x, 0)}{\partial t}, \quad x \in \gamma, \quad[\omega(x)]_{\gamma}=\chi(x, 0), \\
{\left[\sum_{i=1}^{m} \tau_{i}(x, 0) \omega_{x_{i}}(x)+\sigma(x, 0) \omega(x)\right]_{\gamma}=\psi(x, 0), \quad x \in \gamma,} \\
\sum_{i=1}^{m} \nu_{i}(x, 0) \omega_{x_{i}}^{(2)}+\mu(x, 0) \omega^{(2)}(x)=\varphi(x, 0), \quad x \in \partial \Omega . \\
(5) \Omega^{(q)} \in A^{(2, \lambda)}, q=1,2 .
\end{gathered}
$$

We shall now denote by $\mathcal{A}_{l}, \mathcal{A}_{l, \lambda}, \mathcal{B}_{l}, \mathcal{B}_{l, \lambda}$ etc. the sums of the maximum moduli and of the Hölder coefficients of all derivatives of order $l \geq 0$ of the coefficients $a_{i, j}(x, t), b_{i}(x, t)$ etc., respectively.

THEOREM 1.5. Under the above assumptions (1)-(5), for any solution of class $\mathbf{C}^{(2, \lambda)}\left(\overline{Q_{T}}\right)$ of (I) we have

$$
\begin{aligned}
(1.33) \mathcal{U}_{2, \lambda}\left(\bar{Q}_{T}\right)= & \mathcal{O}\left\{\left[\left(1+\mathcal{A}_{0, \lambda}+\mathcal{T}_{0, \lambda}\right)\left(1+\mathcal{B}_{0}+\mathcal{C}_{0}+\mathcal{T}_{1}\right)\right.\right. \\
& +\left(1+\mathcal{A}_{0, \lambda}+\mathcal{N}_{0, \lambda}\right)\left(1+\mathcal{B}_{0}+\mathcal{C}_{0}+\mathcal{N}_{1}\right) \\
& +\left(1+\mathcal{A}_{0, \lambda}+\mathcal{T}_{0, \lambda}+\mathcal{N}_{0, \lambda}\right)^{\frac{1+\lambda}{\lambda}} \\
& \left.+\mathcal{B}_{0, \lambda}+\mathcal{C}_{0, \lambda}+\mathcal{T}_{1, \lambda}+\Sigma_{1, \lambda}+\mathcal{N}_{1, \lambda}+\mathcal{M}_{1, \lambda}\right] \mathcal{F}_{0}
\end{aligned}
$$




$$
\begin{aligned}
& +\left[\left(1+\mathcal{A}_{0, \lambda}+\mathcal{T}_{0, \lambda}\right)\left(1+\mathcal{B}_{0}+\mathcal{T}_{1}\right)\right. \\
& +\left(1+\mathcal{A}_{0, \lambda}+\mathcal{N}_{0, \lambda}\right)\left(1+\mathcal{B}_{0}+\mathcal{N}_{1}\right) \\
& +\left(1+\mathcal{A}_{0, \lambda}+\mathcal{N}_{0, \lambda}\right)^{\frac{1+\lambda}{\lambda}}+\mathcal{C}_{0}+\mathcal{B}_{0, \lambda} \\
& \left.+\mathcal{T}_{1, \lambda}+\mathcal{N}_{1, \lambda}+\Sigma_{1}+\mathcal{M}_{1}\right] \mathcal{F}_{0, \lambda} \\
& +\left(1+\mathcal{A}_{0, \lambda}+\mathcal{T}_{0, \lambda}\right)^{\frac{1+\lambda}{\lambda}} \Psi_{0}+\Psi_{1, \lambda} \\
& +\left(1+\mathcal{A}_{0, \lambda}+\mathcal{N}_{0, \lambda}\right)^{\frac{1+\lambda}{\lambda}} \Phi_{0}+\Phi_{1, \lambda} \\
& +\left[\left(1+\mathcal{A}_{0, \lambda}+\mathcal{T}_{0, \lambda}\right)^{\frac{1+\lambda}{\lambda}}+\left(1+\mathcal{T}_{1}\right)\left(1+\mathcal{A}_{0, \lambda}+\mathcal{T}_{0, \lambda}\right)\right. \\
& \left.+\mathcal{T}_{1, \lambda}+\Sigma_{1, \lambda}\right] \Xi_{0} \\
& +\left[\Sigma_{1}+\left(1+\mathcal{T}_{1}\right)\left(1+\mathcal{A}_{0, \lambda}+\mathcal{T}_{0, \lambda}\right)+\mathcal{T}_{1, \lambda}\right] \Xi_{2, \lambda} \\
& +\left[\left(1+\mathcal{A}_{0, \lambda}+\mathcal{T}_{0, \lambda}+\mathcal{N}_{0, \lambda}\right)^{\frac{1+\lambda}{\lambda}}\right. \\
& +\left(1+\mathcal{B}_{0}+\mathcal{T}_{0, \lambda}+\mathcal{T}_{1}+\mathcal{N}_{1}\right)^{1+\lambda} \\
& +\left(1+\mathcal{A}_{0, \lambda}+\mathcal{T}_{0, \lambda}\right)\left(1+\mathcal{B}_{0}+\mathcal{T}_{1}\right) \\
& +\left(1+\mathcal{A}_{0, \lambda}+\mathcal{N}_{0, \lambda}\right)\left(1+\mathcal{B}_{0}+\mathcal{N}_{1}\right) \\
& \left.+\mathcal{B}_{0, \lambda}+\mathcal{M}_{0, \lambda}+\mathcal{T}_{1, \lambda}+\Sigma_{0, \lambda}+\mathcal{N}_{1, \lambda}\right] \mathcal{U}_{1} \\
& +\left[\left(\mathcal{C}_{0}+\Sigma_{1}+\mathcal{M}_{1}\right)^{1+\lambda / 2}+\left(1+\mathcal{A}_{0, \lambda}+\mathcal{T}_{0, \lambda}+\mathcal{N}_{0, \lambda}\right)^{\frac{1+\lambda}{\lambda}}\right. \\
& \left.+\mathcal{C}_{0}\left(1+\mathcal{A}_{0, \lambda}+\mathcal{T}_{0, \lambda}+\mathcal{N}_{0, \lambda}\right)+\mathcal{C}_{0, \lambda}+\Sigma_{1, \lambda}+\mathcal{M}_{1, \lambda}\right] \mathcal{U}_{0} \\
& +\left[\left(1+\mathcal{A}_{0, \lambda}+\mathcal{T}_{0, \lambda}+\mathcal{N}_{0, \lambda}\right)^{\frac{1+\lambda}{\lambda}}\right. \\
& +\left(1+\mathcal{A}_{0, \lambda}+\mathcal{T}_{0, \lambda}+\mathcal{N}_{0, \lambda}\right)\left(1+\mathcal{B}_{0}+\mathcal{C}_{0}+\mathcal{T}_{1}+\mathcal{N}_{1}\right) \\
& +\mathcal{B}_{0, \lambda}+\mathcal{C}_{0, \lambda}+\mathcal{T}_{1, \lambda}+\mathcal{N}_{1, \lambda}+\mathcal{M}_{1, \lambda} \\
& \left.\left.+\Sigma_{1, \lambda}\right]\left(\mathcal{U}_{(1) 0}+\mathcal{U}_{(1) 0, \lambda}+\Omega_{0}+\Omega_{2, \lambda}\right)\right\},
\end{aligned}
$$

where the estimating constant depends on $m$, the domain $\Omega$ and on the constants from assumptions $(1)-(4)$; the quantities $\mathcal{U}_{(1) 0}$, and $\mathcal{U}_{(1) 0, \lambda}$ relate to the functions $\left.u_{(1)}^{(q)} \equiv \frac{\partial u^{(q)}}{\partial t}\right|_{t=0}, q=1,2$.

1.6. Conjunction problem (see [8]). As an auxiliary problem we consider the conjunction problem for $w(y, t) \in \mathbf{C}_{0}^{(2, \lambda)}\left(\bar{R}_{T}\right)$ that satisfies in the whole space $E_{m+1}$ the parabolic equation with step coefficients and the conjunction conditions on the hyperplane $\Pi_{T}$ :

$$
\left\{\begin{array}{c}
\sum_{i, j=1}^{m} \alpha_{i, j}^{(q)} w_{y_{i} y_{j}}^{(q)}-\frac{\partial w^{(q)}}{\partial t}=h^{(q)}(y, t), \quad(y, t) \in E_{m+1}^{ \pm}, q=1,2, \\
{\left.[w]\right|_{\Pi_{T}}=\eta\left(y^{\prime}, t\right),\left.\quad\left[\sum_{i=1}^{m} \beta_{i} w_{y_{i}}\right]\right|_{\Pi_{T}}=\vartheta\left(y^{\prime}, t\right), \quad\left(y^{\prime}, t\right) \in \Pi_{T},}
\end{array}\right.
$$

where $\alpha_{i, j}^{(q)}, \beta_{i}(i, j=1, \ldots, m, q=1,2)$ are in general complex numbers. 
Lemma 1.6 (see [8, estimate (5.14)]). Let the vector-function $w(y, t) \in$ $\mathbf{C}_{0}^{(2, \lambda)}\left(\bar{R}_{T}\right)$ be a solution of the problem (II). Let the equations of (II) be correctly parabolic and the complementarity condition be fulfilled. Let

$$
h(y, t) \in \stackrel{\circ}{\mathbf{C}}_{0}^{(0, \lambda)}\left(\bar{R}_{T}\right), \quad \eta\left(y^{\prime}, t\right) \in \stackrel{\circ}{C}_{0}^{(2, \lambda)}\left(\Pi_{T}\right), \quad \vartheta\left(y^{\prime}, t\right) \in \stackrel{\circ}{C}_{0}^{(1, \lambda)}\left(\Pi_{T}\right) .
$$

Then we have the a priori estimate

$$
\mathcal{W}_{2, \lambda}\left(\bar{R}_{T}\right)=\mathcal{O}\left(\mathcal{H}_{0, \lambda}+H_{2, \lambda}+\Theta_{1, \lambda}\right)
$$

where the estimating constant depends only on the parabolicity constant, the quantities $A_{0}, B_{0}$ (that relate to $\left.\alpha_{i, j}^{(q)}, \beta_{i}^{(q)}\right), m, \lambda \in(0,1)$ and is independent of $T>0$.

Lemma 1.7. Let the conditions of Lemma 1.6 be fulfilled. Then the problem (II) is uniquely solvable in the class $\stackrel{\circ}{\mathbf{C}}_{0}^{(2, \lambda)}\left(\bar{R}_{T}\right)$.

\section{Reduction of the problem (I) to the problem $(\mathrm{I})_{0}$ with zero initial data}

2.1. Lemma 2.1 (cf. [6, Chapt. IV, Theorem 4.3]). For any vectorfunctions $\omega(x) \in \mathbf{C}^{(2, \lambda)}(\bar{\Omega}), u_{(1)}(x) \in \mathbf{C}^{(0, \lambda)}(\bar{\Omega})$ there is a vector-function $v(x, t) \in \mathbf{C}^{(2, \lambda)}\left(\bar{Q}_{T}\right)$ satisfying

$$
\left.v(x, t)\right|_{t=0}=\omega(x),\left.\quad \frac{\partial v}{\partial t}\right|_{t=0}=u_{(1)}(x), \quad x \in \bar{\Omega} ;
$$

for that function we have the a priori estimates

$$
\begin{aligned}
\mathcal{V}_{0}\left(\bar{Q}_{T}\right) & =\mathcal{O}\left\{\mathcal{U}_{(1) 0}(\bar{\Omega})+\Omega_{0}(\bar{\Omega})+\Omega_{2}(\bar{\Omega})\right\} \\
\mathcal{V}_{2, \lambda}\left(\bar{Q}_{T}\right) & =\mathcal{O}\left\{\mathcal{U}_{(1) 0}(\bar{\Omega})+\mathcal{U}_{(1) 0, \lambda}(\bar{\Omega})+\Omega_{0}(\bar{\Omega})+\Omega_{2, \lambda}(\bar{\Omega})\right\} .
\end{aligned}
$$

Proof. We denote by $\widetilde{\omega}(x), \widetilde{u}_{(1)}(x)$ the extensions of $\omega(x), u_{1}(x)$ onto the whole space $E_{m}$ with the conservation of class (see for example [6, Theorem 4.1, Chapt. IV]): that is, $\widetilde{\omega}(x), \widetilde{u}_{(1)}(x)$ coincide on $\bar{\Omega}$ with $\omega(x), u_{(1)}(x)$ and $\widetilde{\omega}(x) \in \mathbf{C}^{2, \lambda}\left(E_{m}\right), \widetilde{u}_{(1)}(x) \in \mathbf{C}^{0, \lambda}\left(E_{m}\right)$ and also

$$
\begin{aligned}
\|\widetilde{\omega}\|_{\mathbf{C}^{(2, \lambda)}\left(\bar{E}_{m}\right)} & =\mathcal{O}\left\{\|\widetilde{\omega}\|_{\mathbf{C}^{(2, \lambda)}(\bar{\Omega})}\right\}, \\
\left\|\widetilde{u}_{(1)}\right\|_{\mathbf{C}^{(0, \lambda)}\left(\bar{E}_{m}\right)} & =\mathcal{O}\left\{\left\|u_{(1)}\right\|_{\mathbf{C}^{(0, \lambda)}(\bar{\Omega})}\right\} .
\end{aligned}
$$

Now we consider the Cauchy problems in the layer $E_{m} \times[0, T]$ :

$$
\left\{\begin{array}{l}
\frac{\partial w}{\partial t}-\Delta w=0, \quad(x, t) \in E_{m} \times(0, T], \\
\left.w(x, t)\right|_{t=0}=\widetilde{u}_{1}(x)-\Delta \widetilde{\omega}(x), \quad x \in E_{m} .
\end{array}\right.
$$




$$
\left\{\begin{array}{l}
\frac{\partial \widetilde{v}}{\partial t}-\Delta \widetilde{v}=w(x, t), \quad(x, t) \in E_{m} \times(0, T], \\
\left.\widetilde{v}(x, t)\right|_{t=0}=\widetilde{\omega}(x), \quad x \in E_{m} .
\end{array}\right.
$$

It is well known that the problem (2.4) is uniquely solvable in the class $\mathbf{C}^{(0, \lambda)}\left(E_{m} \times[0, T]\right)$ and its solutions satisfy the a priori estimates

$$
\begin{aligned}
\mathcal{W}_{0}\left(E_{m} \times[0, T]\right) & =\mathcal{O}\left\{\widetilde{\mathcal{U}}_{(1) 0}\left(E_{m}\right)+\widetilde{\Omega}_{2}\left(E_{m}\right)\right\}, \\
\mathcal{W}_{0, \lambda}\left(E_{m} \times[0, T]\right) & =\mathcal{O}\left\{\widetilde{\mathcal{U}}_{(1) 0, \lambda}\left(E_{m}\right)+\widetilde{\Omega}_{2, \lambda}\left(E_{m}\right)\right\} .
\end{aligned}
$$

(see [6, Theorem 2.5, Chapt. I and inequality (2.2), Chapt. IV]).

The resulting solution $w(x, t) \in \mathbf{C}^{(0, \lambda)}\left(E_{m} \times[0, T]\right)$ of the problem (2.4) is now substituted in the right hand side of the equation of the problem (2.5). Then by [6, Theorem 5.1, Chapt. IV] the problem (2.5) is uniquely solvable, and its solution $\widetilde{v}(x, t) \in \mathbf{C}^{(2, \lambda)}\left(E_{m} \times[0, T]\right)$ satisfies the a priori estimate

$$
\begin{aligned}
\widetilde{\mathcal{V}}_{2, \lambda}\left(E_{m} \times[0, T]\right)= & \mathcal{O}\left\{\mathcal{W}_{0}\left(E_{m} \times[0, T]\right)+\mathcal{W}_{0, \lambda}\left(E_{m} \times[0, T]\right)\right. \\
& \left.+\widetilde{\Omega}_{0}\left(E_{m}\right)+\widetilde{\Omega}_{2, \lambda}\left(E_{m}\right)\right\} .
\end{aligned}
$$

Moreover, it is well known (see e.g. [6, Theorem 2.5, Chapt. I]) that

$$
\widetilde{\mathcal{V}}_{0}\left(E_{m} \times[0, T]\right)=\mathcal{O}\left\{\mathcal{W}_{0}\left(E_{m} \times[0, T]\right)+\widetilde{\Omega}_{0}\left(E_{m}\right)\right\} .
$$

By (2.4), (2.5), $\left.\frac{\partial \widetilde{v}}{\partial t}\right|_{t=0}=u_{(1)}(x), x \in E_{m}$. Thus we have constructed the vector-function $\widetilde{v}(x, t) \in \mathbf{C}^{(2, \lambda)}\left(E_{m} \times[0, T]\right)$ satisfying

$$
\begin{aligned}
\left.\widetilde{v}(x, t)\right|_{t=0} & =\widetilde{\omega}(x), \\
\left.\frac{\partial \widetilde{v}}{\partial t}\right|_{t=0} & =\widetilde{u}_{(1)}(x), \quad x \in E_{m},
\end{aligned}
$$

and the a priori estimates

$$
\begin{aligned}
\widetilde{\mathcal{V}}_{2, \lambda}\left(E_{m} \times[0, T]\right) & =\mathcal{O}\left\{\widetilde{\mathcal{U}}_{(1) 0}+\widetilde{\mathcal{U}}_{(1) 0, \lambda}+\widetilde{\Omega}_{0}+\widetilde{\Omega}_{2, \lambda}\right\}, \\
\widetilde{\mathcal{V}}_{0}\left(E_{m} \times[0, T]\right) & =\mathcal{O}\left\{\mathcal{U}_{(1) 0}+\widetilde{\Omega}_{0}+\widetilde{\Omega}_{2}\right\},
\end{aligned}
$$

where the quantities on the right hand side relate to the space $E_{m}$. Now we consider some bounded domain $\widetilde{\Omega} \subset \bar{\Omega}$ and the corresponding cylinder $\widetilde{Q}_{T} \subset \bar{Q}_{T}$. We define a cut-off function $\xi(x) \in C^{(2, \lambda)}\left(E_{m}\right)$ such that $0 \leq$ $\xi(x) \leq 1, x \in \widetilde{\Omega}$, and

$$
\xi(x)= \begin{cases}1, & x \in \bar{\Omega}, \\ 0, & x \notin \widetilde{\Omega} .\end{cases}
$$

We consider the vector-function $v(x, t)=\xi(x) \widetilde{v}(x, t)$. By the properties of $\widetilde{v}, \xi$, we derive 


$$
v(x, t) \equiv \begin{cases}\widetilde{v}(x, t), & (x, t) \in \bar{Q}_{T}, \\ 0, & (x, t) \notin \widetilde{Q}_{T},\end{cases}
$$

$v(x, t) \in \mathbf{C}^{(2, \lambda)}\left(E_{m} \times[0, T]\right)$ and

$$
\begin{aligned}
\mathcal{V}_{2, \lambda}\left(E_{m} \times[0, T]\right) & =\mathcal{O}\left\{\widetilde{\mathcal{V}}_{2, \lambda}\left(E_{m} \times[0, T]\right)+\widetilde{\mathcal{V}}_{0}\left(E_{m} \times[0, T]\right)\right\}, \\
\mathcal{V}_{0}\left(E_{m} \times[0, T]\right) & \leq \widetilde{\mathcal{V}}_{0}\left(E_{m} \times[0, T]\right)
\end{aligned}
$$

From (2.9)-(2.11) with regard to (2.3) we finally obtain the desired a priori estimates $(2.1),(2.2)$. From the properties of $\widetilde{v}(x, t), \widetilde{\omega}(x) \widetilde{u}_{(1)}(x)$ it is easy to see that the constructed vector-function $v(x, t)$ satisfies the initial conditions of the lemma.

2.2. Now we are in a position to reduce the problem (I) to the boundary value problem with zero initial data. Following $[6,8]$ we write

$$
u_{(k)}^{(q)}(x)=\left.\frac{\partial^{k} u^{(q)}(x, t)}{\partial t^{k}}\right|_{t=0}, \quad k=0,1, q=1,2 .
$$

If $u(x, t)$ is a solution of the boundary value problem (I), then we have

$$
\begin{aligned}
u_{(0)}^{(q)}(x)= & \omega^{(q)}(x), \quad x \in \overline{\Omega^{(q)}}, q=1,2, \\
u_{(1)}^{(q)}(x)= & \mathcal{L}^{(q)}\left(x, 0, D_{x}\right) \omega^{(q)}(x)-f^{(q)}(x, 0) \\
\equiv & \sum_{i, j=1}^{m} a_{i, j}^{(q)}(x, 0) \omega_{x_{i} x_{j}}^{(q)}(x)+\sum_{i=1}^{m} b_{i}^{(q)}(x, 0) \omega_{x_{i}}^{(q)}(x) \\
& +c^{(q)}(x, 0) \omega^{(q)}(x)-f^{(q)}(x, 0), \quad x \in \overline{\Omega^{(q)}}, q=1,2 .
\end{aligned}
$$

Let $v(x, t)$ be the vector-function constructed in Lemma 2.1. Then

$$
w(x, t)=u(x, t)-v(x, t)
$$

is a solution of the boundary value problem

$$
\left\{\begin{array}{l}
\mathcal{L}^{(q)}\left(x, t, D_{x}\right) w^{(q)}=\frac{\partial w^{(q)}}{\partial t}+\widetilde{f}^{(q)}(x, t), \quad(x, t) \in Q_{T}^{(q)}, q=1,2, \\
{[w]_{\gamma_{T}}=\widetilde{\chi}(x, t), \quad\left[\sum_{i=1}^{m} \tau_{i}(x, t) w_{x_{i}}+\sigma(x, t) w\right]_{\gamma_{T}}=\widetilde{\psi}(x, t), \quad(x, t) \in \gamma_{T},} \\
\sum_{i=1}^{m} \nu_{i}(x, t) w_{x_{i}}^{(2)}+\left.\mu(x, t) w^{(2)}\right|_{\partial_{T} \Omega}=\widetilde{\varphi}(x, t), \quad(x, t) \in \partial_{T} \Omega, \\
w^{(q)}(x, 0)=0,\left.\quad \frac{\partial w^{(q)}}{\partial t}\right|_{t=0}=0, \quad x \in \overline{\Omega^{(q)}}, q=1,2,
\end{array}\right.
$$


where

$$
\begin{aligned}
\widetilde{f}^{(q)}(x, t) & =f^{(q)}(x, t)-\mathcal{L}^{(q)} v^{(q)}+\frac{\partial v^{(q)}}{\partial t}, \quad q=1,2, \\
\widetilde{\chi}(x, t) & =\chi(x, t)-[v]_{\gamma_{T}}, \\
\widetilde{\psi}(x, t) & =\psi(x, t)-\left[\sum_{i=1}^{m} \tau_{i}(x, t) v_{x_{i}}+\sigma(x, t) v\right]_{\gamma_{T}}, \\
\widetilde{\varphi}(x, t) & =\varphi(x, t)-\left.\left\{\sum_{i=1}^{m} \nu_{i}(x, t) v_{x_{i}}^{(2)}+\mu(x, t) v^{(2)}\right\}\right|_{\partial_{T} \Omega} .
\end{aligned}
$$

By the properties of $v(x, t),(2.12)$ and the compatibility conditions (4) from Assumptions I it is not difficult to derive that

$$
\begin{aligned}
& \tilde{f} \in \mathbf{C}_{0}^{(2, \lambda)}\left(\bar{Q}_{T}\right), \quad \widetilde{\psi} \in C_{0}^{(1, \lambda)}\left(\gamma_{T}\right), \\
& \tilde{\varphi} \in C_{0}^{(1, \lambda)}\left(\partial_{T} \Omega\right), \quad \tilde{\chi} \in C_{0}^{(2, \lambda)}\left(\gamma_{T}\right) .
\end{aligned}
$$

The equalities $(\mathrm{I})_{0}$ together with conditions $(2.15)$ will be called the boundary value problem with zero initial data.

Thus, as (2.13) shows, for the proof of Theorem 1.5 it is necessary to investigate the boundary value problem $(\mathrm{I})_{0}$ and to derive the corresponding a priori estimates for its solutions.

2.3. Calculating the quantities for the function $u_{(1)}(x)$ from $(2.12)$ we now obtain, by the estimates (2.1), (2.2),

$$
\begin{aligned}
\mathcal{V}_{0}= & \mathcal{O}\left\{\mathcal{F}_{0}+\left(1+\mathcal{B}_{0}+\mathcal{C}_{0}\right) \Omega_{0}+\left(1+\mathcal{B}_{0}\right) \Omega_{2}\right\} \\
\mathcal{V}_{2, \lambda}= & \mathcal{O}\left\{\mathcal{F}_{0}+\mathcal{F}_{0, \lambda}+\left(1+\mathcal{A}_{0, \lambda}+\mathcal{B}_{0}+\mathcal{C}_{0}+\mathcal{B}_{0, \lambda}+\mathcal{C}_{0, \lambda}\right) \Omega_{0}\right. \\
& \left.+\left(1+\mathcal{A}_{0, \lambda}+\mathcal{B}_{0}+\mathcal{C}_{0}+\mathcal{B}_{0, \lambda}\right) \Omega_{2, \lambda}\right\}
\end{aligned}
$$

3. Local estimates of solutions of the problem $(\mathrm{I})_{0}$. Now we derive local estimates of solutions of $(\mathrm{I})_{0}$. We shall consider cylinders of type $(1)$ from Section 1.4. The estimates for the other cylinders are derived similarly.

Let $\varrho>r\left(P_{0}, \sigma\right)$ and let $c_{\tau}^{(\varkappa)}, c_{\tau}^{(k \varkappa)}$ be cylinders related to the surfaces $\widetilde{\Delta}^{(q)}, \sigma_{\tau}$. Let $w(x, t) \in \mathbf{C}^{(2, \lambda)}\left(\bar{Q}_{T}\right)$ be a solution of $(\mathrm{I})_{0}$ and first suppose that

$$
b_{i}^{(q)}(x, t) \equiv c^{(q)}(x, t) \equiv \sigma(x, t) \equiv 0, \quad i=1, \ldots, m, q=1,2 .
$$

Since we are considering only a neighbourhood of $\gamma_{T}$, no attention need be given to the boundary condition on $\partial_{T} \Omega$. On carrying out the coordinate transformation (1.4) we find that $\bar{w}(y, t)$ (the image of $w(x, t)$ ) satisfies the 
conjunction problem

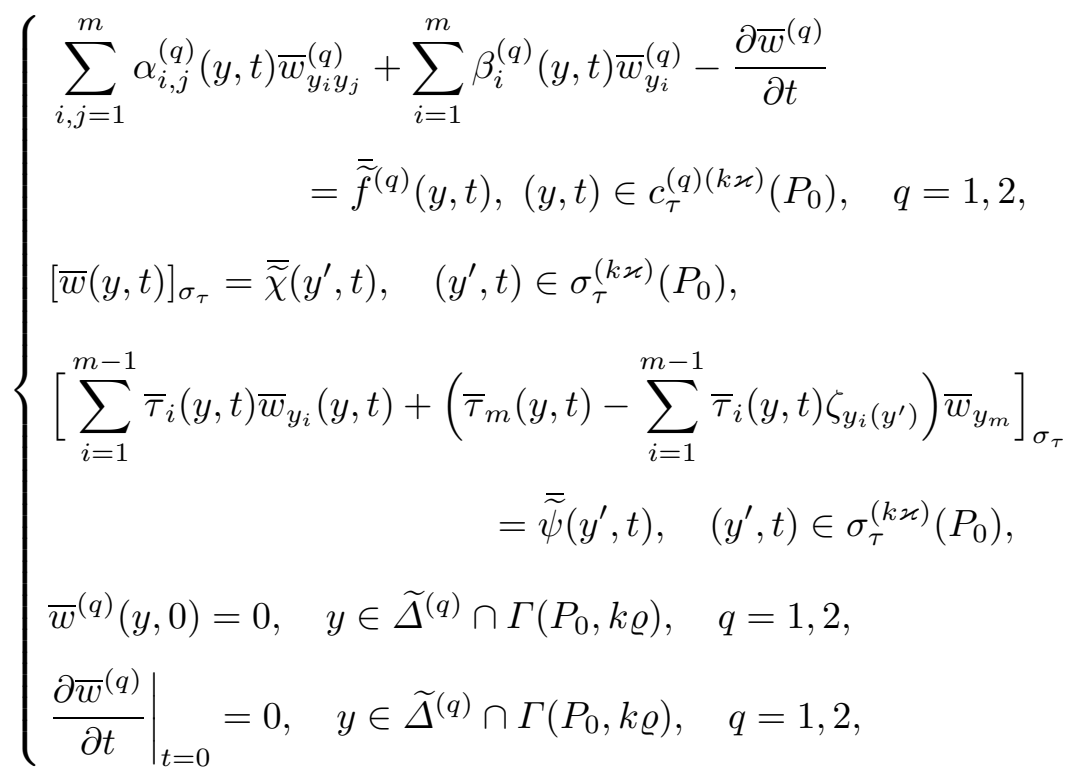

where the functions $\alpha_{i, j}^{(q)}(y, t), \beta_{i}^{(q)}(y, t)$ depend on $y, t$ through $\bar{a}_{i, j}^{(q)}(y, t)$ and the first and second derivatives of $\zeta(y)$ from (1.4).

We put

$$
\begin{aligned}
& {\left[\bar{\tau}_{i}(y, t)\right]_{\sigma_{\tau}}=l_{i}\left(y^{\prime}, t\right), \quad i=1, \ldots, m-1,} \\
& \bar{\tau}_{m}^{(q)}(y, t)-\sum_{i=1}^{m-1} \bar{\tau}_{i}^{(q)}(y, t) \zeta_{y_{i}}\left(y^{\prime}\right)=l_{m}^{(q)}(y, t), \quad q=1,2,
\end{aligned}
$$

and

$$
\begin{aligned}
g^{(q)}(y, t) \equiv \overline{\widetilde{f}}^{(q)}(y, t)-\sum_{i, j=1}^{m}\left(\alpha_{i, j}^{(q)}(y, t)-\alpha_{i, j}^{(q)}\left(y_{0}, 0\right)\right) \bar{w}_{y_{i} y_{j}}^{(q)} \\
-\sum_{i=1}^{m} \beta_{i}^{(q)}(y, t) \bar{w}_{y_{i}}^{(q)}, \quad q=1,2, \\
\theta\left(y^{\prime}, t\right) \equiv \overline{\widetilde{\psi}}\left(y^{\prime}, t\right)-\left[\sum_{i=1}^{m-1}\left(\bar{\tau}_{i}(y, t)-\bar{\tau}_{i}\left(y_{0}, 0\right)\right) \bar{w}_{y_{i}}(y, t)\right]_{\sigma_{\tau}} \\
-\left[\left(l_{m}(y, t)-l_{m}\left(y_{0}, 0\right)\right) \bar{w}_{y_{m}}\right]_{\sigma_{\tau}} .
\end{aligned}
$$

Then the problem (3.2) can be put in the form 


$$
\left\{\begin{array}{l}
\sum_{i, j=1}^{m} \alpha_{i, j}^{(q)}\left(y_{0}, 0\right) \bar{w}_{y_{i} y_{j}}^{(q)}-\frac{\partial \bar{w}^{(q)}}{\partial t}=g^{(q)}(y, t), \quad(y, t) \in c_{\tau}^{(q)(k \varkappa)}\left(P_{0}\right), \quad q=1,2, \\
{[\bar{w}(y, t)]_{\sigma_{\tau}}=\overline{\widetilde{\chi}}\left(y^{\prime}, t\right), \quad\left[\frac{\partial \bar{w}}{\partial l}\right]_{\sigma_{\tau}}=\theta\left(y^{\prime}, t\right), \quad\left(y^{\prime}, t\right) \in \sigma_{\tau}^{(k \varkappa)}\left(P_{0}\right),} \\
\bar{w}^{(q)}(y, 0)=0,\left.\quad \frac{\partial \bar{w}^{(q)}}{\partial t}\right|_{t=0}=0, \quad y \in \widetilde{\Delta}^{(q)} \cap \Gamma\left(P_{0}, k \varrho\right), \quad q=1,2,
\end{array}\right.
$$

where $l$ is the axis with direction cosines

$$
\left\{\bar{\tau}_{1}\left(y_{0}^{\prime}, 0\right), \ldots, \bar{\tau}_{m-1}\left(y_{0}^{\prime}, 0\right), l_{m}\left(y_{0}^{\prime}, 0\right)\right\} .
$$

Our aim is to find an estimate for $\overline{\mathcal{W}}_{(\tau) 2, \lambda}^{(\varkappa)}$. To this end we define the new vector-function $z(x, t)=\xi(y) \bar{w}(y, t)$, where $\xi(y)$ is the truncation function

$$
\xi(y)= \begin{cases}1, & y \in \widetilde{\Delta} \cap \Gamma\left(P_{0}, \varrho\right), \\ 0, & y \notin \widetilde{\Delta} \cap \Gamma\left(P_{0}, k \varrho\right),\end{cases}
$$

with the properties

(1) $0 \leq \xi(y) \leq 1$,

(2) $D^{n} \xi=\mathcal{O}\left(\varrho^{-n}\right) \forall n \in \mathbb{N}$.

The vector-function $z(y, t)$ is a solution of the problem

$$
\left\{\begin{array}{l}
\sum_{i, j=1}^{m} \alpha_{i, j}^{(q)}\left(y_{0}, 0\right) z_{y_{i} y_{j}}^{(q)}-\frac{\partial z^{(q)}}{\partial t}=h^{(q)}(y, t), \\
\quad(y, t) \in c_{\tau}^{(q)(k \varkappa)}\left(P_{0}\right), q=1,2, \\
{[z]_{\sigma_{\tau}}=\eta\left(y^{\prime}, t\right), \quad\left[\frac{\partial z}{\partial l}\right]_{\sigma_{\tau}}=\vartheta\left(y^{\prime}, t\right), \quad\left(y^{\prime}, t\right) \in \sigma_{\tau}^{(k \varkappa)}\left(P_{0}\right),} \\
z^{(q)}(y, 0)=0,\left.\quad \frac{\partial z^{(q)}}{\partial t}\right|_{t=0}=0, \quad y \in E_{m}, q=1,2,
\end{array}\right.
$$

where $h^{(q)}(y, t), \eta\left(y^{\prime}, t\right), \vartheta\left(y^{\prime}, t\right)$ are defined by $[1,(2.17)]$; moreover, it is not difficult to verify by $(2.15)$ that

$$
\begin{aligned}
& h(y, t) \in \stackrel{\circ}{\mathbf{C}}_{0}^{(0, \lambda)}\left(\bar{R}_{\tau}\right), \quad \eta\left(y^{\prime}, t\right) \in \stackrel{\circ}{C}_{0}^{(2, \lambda)}\left(\Pi_{\tau}\right), \\
& \vartheta\left(y^{\prime}, t\right) \in \stackrel{\circ}{C}_{0}^{(1, \lambda)}\left(\Pi_{\tau}\right) .
\end{aligned}
$$

Therefore the vector-function $z(y, t)$ is in $\stackrel{\mathbf{C}}{0}^{(2, \lambda)}\left(\bar{R}_{\tau}\right)$ and is a solution of the problem (3.6), a problem of type (II); moreover, (3.7) and Assumptions I guarantee the fulfilment of all conditions of Lemmas 1.6 and 1.7. Hence the problem (3.6) has a unique solution $z(y, t)$ of class $\stackrel{\mathbf{C}}{0}^{(2, \lambda)}\left(\bar{R}_{\tau}\right)$ and the estimate

$$
\mathcal{Z}_{(\tau) 2, \lambda}^{(k \varkappa)}=\mathcal{O}\left(\mathcal{H}_{(\tau) 0, \lambda}^{(k \varkappa)}+H_{(\tau) 2, \lambda}^{(\nu)}+\Theta_{(\tau) 1, \lambda}^{(\nu)}\right)
$$


holds uniformly with respect to $\tau$. Now taking account of the trivial inequality

$$
\overline{\mathcal{W}}_{(\tau) 2, \lambda}^{(\varkappa)} \equiv \mathcal{Z}_{(\tau) 2, \lambda}^{(\varkappa)} \leq \mathcal{Z}_{(\tau) 2, \lambda}^{(k \varkappa)}, \quad k>3
$$

and calculating the quantities on the right side of (3.8) (see [1, p. 23]) we obtain, by (3.8)-(3.9),

$$
\begin{aligned}
\overline{\mathcal{W}}_{(\tau) 2, \lambda}^{(\varkappa)}= & \mathcal{O}\left(\varepsilon \overline{\mathcal{W}}_{(\tau) 2, \lambda}^{(k \varkappa)}+\varrho^{-(2+\lambda)} \overline{\mathcal{W}}_{(\tau) 0}^{(k \varkappa)}+\mathcal{G}_{(\tau) 0, \lambda}^{(k \varkappa)}+\varrho^{-\lambda} \mathcal{G}_{(\tau) 0}^{(k \varkappa)}\right. \\
& \left.+\overline{\widetilde{\Xi}}_{(\tau) 2, \lambda}^{(\nu)}+\Theta_{(\tau) 1, \lambda}^{(\nu)}+\varrho^{-(1+\lambda)} \Theta_{(\tau) 0}^{(\nu)}\right), \quad \forall \varepsilon>0
\end{aligned}
$$

(here we use the inequality (1.10) of Lemma 1.1). This is the desired estimate. It should be noted that the $\mathcal{O}$-quantity on the right side of (3.10) depends only on $\mathcal{A}_{0}+\mathcal{T}_{0}=\mathcal{O}(1)$ (see condition 2) of Assumptions I), the parabolicity constant, and the numbers $m, \lambda$, but it is independent of $\tau$. The estimate (3.10) may be simplified (cf. [1]). In fact, the vector-function $\bar{w}(y, t)=\left(\bar{w}^{(1)}(y, t), \bar{w}^{(2)}(y, t)\right)$ can be decomposed by the Taylor formula in the neighbourhood of $P_{0}^{\prime}=\left(y_{0}^{\prime}, 0,0\right) \in \sigma$ with zero initial data:

$$
\begin{aligned}
w^{(q)}(y, t)= & \bar{w}^{(q)}\left(P_{0}^{\prime}\right)+\sum_{i=1}^{m-1}\left(y_{i}-y_{0 i}^{\prime}\right) \bar{w}_{y_{i}}^{(q)}\left(\widetilde{y}^{\prime}, 0,0\right) \\
& +y_{m} \bar{w}_{y_{m}}^{(q)}\left(y_{0}^{\prime}, \widetilde{y}_{m}, 0\right)+t \bar{w}_{t}^{(q)}\left(y_{0}^{\prime}, 0, \widetilde{t}\right) \\
= & t \bar{w}_{t}^{(q)}\left(y_{0}^{\prime}, 0, \widetilde{t}\right), \\
& (\widetilde{y}, \widetilde{t}) \in c_{\tau}^{(q)(k \varkappa)} \cap \Gamma_{m+1}\left(P_{0}^{\prime}, r\left(P, P_{0}^{\prime}\right)\right), q=1,2 .
\end{aligned}
$$

Now we choose $\tau=\varrho^{2}$. By (3.11) we obtain

$$
\overline{\mathcal{W}}_{0}^{(k \varkappa)}=\mathcal{O}\left(\tau \overline{\mathcal{W}}_{2}^{(k \varkappa)}\right)=\mathcal{O}\left(\varrho^{2} \overline{\mathcal{W}}_{2}^{(k \varkappa)}\right)
$$

Further, it is easy to see by (2.15) and initial data of (3.5) from (3.4) that $\theta\left(y^{\prime}, 0\right)=0$ and therefore we have

$$
\begin{aligned}
\theta\left(y^{\prime}, t\right) & =\left(\theta\left(y^{\prime}, t\right)-\theta\left(y_{0}^{\prime}, t\right)\right)+\left(\theta\left(y_{0}^{\prime}, t\right)-\theta\left(y_{0}^{\prime}, 0\right)\right) \\
& =\mathcal{O}\left\{\left|y^{\prime}-y_{0}^{\prime}\right| \Theta_{1}^{(\nu)}+\tau^{\frac{1+\lambda}{2}} \Theta_{1, \lambda}^{(\nu)}\right\} \\
& =\mathcal{O}\left(\varrho \Theta_{1}^{(\nu)}+\varrho^{1+\lambda} \Theta_{1, \lambda}^{(\nu)}\right) .
\end{aligned}
$$

Thus by (3.12), (3.13) the estimate (3.10) simplifies to

$$
\begin{aligned}
\overline{\mathcal{W}}_{2, \lambda}^{(\varkappa)}= & \mathcal{O}\left(\varepsilon \overline{\mathcal{W}}_{2, \lambda}^{(k \varkappa)}+\varrho^{-\lambda} \overline{\mathcal{W}}_{2}^{(k \varkappa)}+\mathcal{G}_{0, \lambda}^{(k \varkappa)}+\varrho^{-\lambda} \mathcal{G}_{0}^{(k \varkappa)}+\overline{\widetilde{\Xi}}_{2, \lambda}^{(\nu)}\right. \\
& \left.+\Theta_{1, \lambda}^{(\nu)}+\varrho^{-\lambda} \Theta_{1}^{(\nu)}\right), \quad \forall \varepsilon>0 .
\end{aligned}
$$

Calculating the quantities on the right side of (3.14) in the same way as in [1] and setting $\varepsilon=\varrho^{\lambda}$ we see that for any solution of (3.2) we have the 
estimate (cf. $[1,(2.25)])$

$$
\begin{aligned}
\overline{\mathcal{W}}_{2, \lambda}^{(\varkappa)}= & \mathcal{O}\left\{\overline{\widetilde{\mathcal{F}}}_{0, \lambda}^{(k \varkappa)}+\varrho^{-\lambda}\left(\overline{\widetilde{\mathcal{F}}}_{0}^{(k \varkappa)}+\overline{\widetilde{\Psi}}_{1}^{(\nu)}\right)\right. \\
& +\left(1+\overline{\mathcal{T}}_{0, \lambda}^{(\nu)}+\overline{\mathcal{T}}_{1}^{(\nu)}+\varrho^{-\lambda} \overline{\mathcal{T}}_{1}^{(\nu)}+\overline{\mathcal{T}}_{1, \lambda}^{(\nu)}\right)\left(\overline{\widetilde{\Xi}}_{0}^{(\nu)}+\overline{\widetilde{\Xi}}_{2, \lambda}^{(\nu)}\right) \\
& +\varrho^{\lambda}\left(1+\overline{\mathcal{A}}_{0, \lambda}^{(k \varkappa)}+\overline{\mathcal{T}}_{0, \lambda}^{(\nu)} \overline{\mathcal{W}}_{2, \lambda}^{(k \varkappa)}\right. \\
& +\left(1+\varrho^{-\lambda}+\overline{\mathcal{A}}_{0, \lambda}^{(k \varkappa)}+\overline{\mathcal{T}}_{0, \lambda}^{(\nu)}\right) \overline{\mathcal{W}}_{2}^{(k \varkappa)} \\
& +\left(1+\overline{\mathcal{T}}_{0, \lambda}^{(\nu)}+\overline{\mathcal{T}}_{1}^{(\nu)}\right) \overline{\mathcal{W}}_{1, \lambda}^{(k \varkappa)}+\overline{\widetilde{\Psi}}_{1, \lambda}^{(\nu)} \\
& \left.+\left(1+\varrho^{-\lambda}+\overline{\mathcal{A}}_{0, \lambda}^{(k \varkappa)}+\overline{\mathcal{T}}_{0, \lambda}^{(\nu)}+\overline{\mathcal{T}}_{1}^{(\nu)}+\varrho^{-\lambda} \overline{\mathcal{T}}_{1}^{(\nu)}+\overline{\mathcal{T}}_{1, \lambda}^{(\nu)}\right) \overline{\mathcal{W}}_{1}^{(k \varkappa)}\right\} .
\end{aligned}
$$

We put

$$
\mathcal{J} \equiv 1+\overline{\mathcal{A}}_{0, \lambda}+\overline{\mathcal{T}}_{0, \lambda}
$$

and rewrite (3.15) in shortened form:

$$
\overline{\mathcal{W}}_{2, \lambda}^{(\varkappa)}=\mathcal{O}\left(\varrho^{\lambda} \mathcal{J}^{(k \varkappa)} \overline{\mathcal{W}}_{2, \lambda}^{(k \varkappa)}+\mathcal{K}\left(\varkappa ; P_{0}\right)\right) .
$$

We multiply both sides of (3.17) by $\varrho^{2+\lambda}$ and take into account the inequality (1.29) of Lemma 1.3:

$$
\begin{array}{r}
\varrho^{2+\lambda} \overline{\mathcal{W}}_{2, \lambda}^{(\varkappa)} \leq k_{1} \varrho_{0}^{\lambda} \mathcal{J}\left(\widetilde{p}_{\tau}\right)\left(\overline{\mathcal{B}}_{2, \lambda}^{(\varkappa)}+\overline{\mathcal{B}}_{2}^{(\varkappa)}\right)+k_{2} \varrho_{0}^{2+\lambda} \mathcal{K}\left(\varkappa, P_{0}\right), \\
\forall \tau \geq d_{0}^{2}, \varrho_{0}=\varkappa d_{0},
\end{array}
$$

where $k_{1}, k_{2}$ are constants independent of $\varkappa, \tau$. Up to this point the number $\varkappa$ was any positive number such that $\varkappa \leq \varkappa_{0}<1, k \varkappa<4, k>3$. Now let

$$
\varkappa_{1}=\sup \left\{\varkappa \mid \varkappa<\varkappa_{0}, k_{1} \varrho_{0}^{\lambda} \mathcal{J}\left(\widetilde{p}_{\tau}\right)<1 / 4\right\} .
$$

Then we derive

$$
\left(\varkappa_{1} d_{0}\right)^{-\lambda}=\mathcal{O}\left(1+\mathcal{J}\left(\widetilde{p}_{\tau}\right)\right) .
$$

Now fix the point $P_{0}$ so that

$$
\left(\varkappa_{1} d\left(P_{0}\right)\right)^{2+\lambda} \overline{\mathcal{W}}_{2, \lambda}^{\left(\varkappa_{1}\right)}>\frac{1}{2} \overline{\mathcal{B}}_{2, \lambda}^{\left(\varkappa_{1}\right)} .
$$

Then from (3.18), (3.19) we obtain

$$
\overline{\mathcal{B}}_{2, \lambda}^{\left(\varkappa_{1}\right)}=\mathcal{O}\left(\overline{\mathcal{B}}_{2}^{\left(\varkappa_{1}\right)}\left(\widetilde{p}_{\tau}\right)+\left(\varkappa_{1} d\left(P_{0}\right)\right)^{2+\lambda} \mathcal{K}\left(\varkappa_{1} ; \widetilde{p}_{\tau}\right)\right) .
$$

Let $\pi_{\tau}$ be cylinders that are symmetric with respect to the hyperplane $\sigma_{\tau}$ and satisfy the assumptions of Lemma 1.4. By this lemma with $\varkappa=\varkappa_{1}$ and the inequality (3.21) we have

$$
\delta^{2+\lambda} \overline{\mathcal{W}}_{2, \lambda}\left(\pi_{\tau}\right)=\mathcal{O}\left(\left(\varkappa_{1} d_{0}\right)^{-\lambda} \overline{\mathcal{W}}_{2}\left(\widetilde{p}_{\tau}\right)+\mathcal{K}\left(\varkappa_{1} ; \widetilde{p}_{\tau}\right)\right), \quad \pi_{\tau} \subset \widetilde{p}_{\tau}
$$


Hence taking into account $(3.20)$ and recalling the expression for $\mathcal{K}\left(\varkappa_{1} ; \widetilde{p}_{\tau}\right)$ from (3.17) and (3.15) we finally obtain

$$
\begin{aligned}
\delta^{2+\lambda} \overline{\mathcal{W}}_{2, \lambda}\left(\pi_{\tau}\right)= & \mathcal{O}\left\{\overline{\widetilde{\mathcal{F}}}_{0, \lambda}+\mathcal{J} \overline{\mathcal{F}}_{0}\right. \\
& +\left(1+\overline{\mathcal{T}}_{0, \lambda}+\mathcal{J} \overline{\mathcal{T}}_{1}+\overline{\mathcal{T}}_{1, \lambda}\right)\left(\overline{\widetilde{\Xi}}_{0}+\overline{\widetilde{\Xi}}_{2, \lambda}\right) \\
& +\overline{\widetilde{\Psi}}_{1, \lambda}+\mathcal{J} \overline{\widetilde{\Psi}}_{1}+\mathcal{J} \overline{\mathcal{W}}_{2}+\left(1+\overline{\mathcal{T}}_{0, \lambda}+\overline{\mathcal{T}}_{1}\right) \overline{\mathcal{W}}_{1, \lambda} \\
& \left.+\left(\mathcal{J}\left(1+\overline{\mathcal{T}}_{1}\right)+\overline{\mathcal{T}}_{1, \lambda}\right) \overline{\mathcal{W}}_{1}\right\}
\end{aligned}
$$

where the quantities on the right hand side relate to the cylinder $\widetilde{p}_{\tau} \supset \pi_{\tau}$.

On applying the transformation inverse to (1.4) the cylinders $\pi_{\tau}^{(q)}$ go to cylinders $\Pi_{\tau}^{(q)} \subset Q_{* \tau}^{(q)}$, whose boundary contains the part of $\gamma_{* \tau} \cup \Omega_{*}^{(q)}$ and whose distance from $\partial\left[Q_{* \tau}^{(q)} \backslash\left(\gamma_{* \tau} \cup \Omega_{*}^{(q)}\right)\right]$, taken to be minimum over $q=1,2$ and denoted by $\delta$, is positive. By (3.16) we now have

$$
\begin{aligned}
\delta^{2+\lambda} \mathcal{W}_{2, \lambda}\left(\Pi_{\tau}\right)= & \mathcal{O}\left\{\left(1+\mathcal{A}_{0, \lambda}+\mathcal{T}_{0, \lambda}\right)\left(\widetilde{\mathcal{F}}_{0}+\widetilde{\Psi}_{1}\right)\right. \\
& +\left\{1+\mathcal{T}_{0, \lambda}+\left(1+\mathcal{A}_{0, \lambda}+\mathcal{T}_{0, \lambda}\right) \mathcal{T}_{1}+\mathcal{T}_{1, \lambda}\right\}\left(\widetilde{\Xi}_{0}+\widetilde{\Xi}_{2, \lambda}\right) \\
& +\left(1+\mathcal{A}_{0, \lambda}+\mathcal{T}_{0, \lambda}\right) \mathcal{W}_{2}+\left(1+\mathcal{T}_{0, \lambda}+\mathcal{T}_{1}\right) \mathcal{W}_{1, \lambda} \\
& +\widetilde{\mathcal{F}}_{0, \lambda}+\widetilde{\Psi}_{1, \lambda} \\
& \left.+\left\{\left(1+\mathcal{A}_{0, \lambda}+\mathcal{T}_{0, \lambda}\right)\left(1+\mathcal{T}_{1}\right)+\mathcal{T}_{1, \lambda}\right\} \mathcal{W}_{1}\right\},
\end{aligned}
$$

where the quantities on the right side relate to the cylinder $Q_{* \tau}$.

If we now discard the assumption (3.1), we must use the estimate (3.23), replacing $\widetilde{f}^{(q)}(x, t)$ by the function

$$
\widetilde{f}^{(q)}(x, t)-\sum_{i=1}^{m} b_{i}^{(q)}(x, t) w_{x_{i}}^{(q)}-c^{(q)}(x, t) w^{(q)}
$$

and $\widetilde{\psi}(x, t)$ by $\widetilde{\psi}(x, t)-[\sigma(x, t) w]_{\gamma_{\tau}}$.

Therefore for the solution of the problem $(\mathrm{I})_{0}$ we arrive at the following local estimate in the neighborhood of the intersection $\Omega \cap \gamma_{T}$ :

$$
\begin{aligned}
& \delta^{2+\lambda} \mathcal{W}_{2, \lambda}\left(\Pi_{\tau}\right) \\
& =\mathcal{O}\left\{\left\{1+\mathcal{T}_{0, \lambda}+\left(1+\mathcal{A}_{0, \lambda}+\mathcal{T}_{0, \lambda}\right)\left(\mathcal{T}_{1}+\Sigma_{0}\right)+\mathcal{T}_{1, \lambda}+\Sigma_{1}\right\} \widetilde{\Xi}_{2, \lambda}\right. \\
& \quad+\left\{1+\mathcal{T}_{0, \lambda}+\left(1+\mathcal{A}_{0, \lambda}+\mathcal{T}_{0, \lambda}\right)\left(\mathcal{T}_{1}+\Sigma_{0}+\Sigma_{1}\right)+\mathcal{T}_{1, \lambda}+\Sigma_{1, \lambda}\right\} \widetilde{\Xi}_{0} \\
& \quad+\left(1+\mathcal{A}_{0, \lambda}+\mathcal{T}_{0, \lambda}\right) \mathcal{W}_{2}+\left(1+\mathcal{T}_{0, \lambda}+\mathcal{T}_{1}+\mathcal{B}_{0}+\mathcal{S}_{0}\right) \mathcal{W}_{1, \lambda} \\
& \quad+\left\{\left(1+\mathcal{A}_{0, \lambda}+\mathcal{T}_{0, \lambda}\right)\left(1+\mathcal{T}_{1}+\mathcal{B}_{0}+\mathcal{S}_{0}\right)+\mathcal{T}_{1, \lambda}+\mathcal{B}_{0, \lambda}+\mathcal{S}_{0, \lambda}\right\} \mathcal{W}_{1} \\
& \quad+\left\{\left(\mathcal{C}_{0}+\mathcal{S}_{1}\right)\left(1+\mathcal{A}_{0, \lambda}+\mathcal{T}_{0, \lambda}\right)+\mathcal{C}_{0, \lambda}+\mathcal{S}_{1, \lambda}\right\} \mathcal{W}_{0} \\
& \left.\quad+\left(1+\mathcal{A}_{0, \lambda}+\mathcal{T}_{0, \lambda}\right)\left(\widetilde{\mathcal{F}}_{0}+\widetilde{\Psi}_{1}\right)+\left(\mathcal{C}_{0}+\mathcal{S}_{1}\right) \mathcal{W}_{0, \lambda}+\widetilde{\mathcal{F}}_{0, \lambda}+\widetilde{\Psi}_{1, \lambda}\right\},
\end{aligned}
$$


where the quantities on the right side relate to the cylinder $Q_{* \tau}$; here the $\mathcal{S}$-quantities relate to the function $s(x, t)=[\sigma(x, t)]_{\gamma_{\tau}}$. We note that the estimate (3.24) is uniform with respect to $\tau$.

4. Conditional existence theorem. In this section we consider the nonlinear problem

$$
\left\{\begin{array}{l}
L^{(q)}\left(x, t, D_{x}\right) u^{(q)}=0, \quad(x, t) \in Q_{T}^{(q)}, \\
{[u]_{\gamma_{T}}=\chi(x, t),} \\
{\left[\Lambda^{\gamma_{T}} u\right] \equiv\left[b\left(x, t, u, u_{x}\right)+\sum_{i=1}^{m} b_{i}(x, t, u) u_{x_{i}}+b_{0}(x, t, u)\right]_{\gamma_{T}}} \\
=\psi(x, t), \quad(x, t) \in \gamma_{T}, \\
\Lambda^{\partial_{T} \Omega} u \equiv c\left(x, t, u^{(2)}, u_{x}^{(2)}\right)+\sum_{i=1}^{m} c_{i}\left(x, t, u^{(2)}\right) u_{x_{i}}^{(2)}+\left.c_{0}\left(x, t, u^{(2)}\right)\right|_{\partial_{T} \Omega} \\
=\varphi(x, t), \quad(x, t) \in \partial_{T} \Omega, \\
u^{(q)}(x, 0)=\omega^{(q)}(x), \quad x \in \overline{\Omega^{(q)}}, q=1,2,
\end{array}\right.
$$

where

$$
\begin{aligned}
L^{(q)}\left(x, t, D_{x}\right) u^{(q)} \equiv & \sum_{i, j=1}^{m} a_{i, j}^{(q)}\left(x, t, u^{(q)}, u_{x}^{(q)}\right) u_{x_{i} x_{j}}^{(q)}+a^{(q)}\left(x, t, u^{(q)}, u_{x}^{(q)}\right) \\
& -\frac{\partial u^{(q)}}{\partial t} .
\end{aligned}
$$

For this problem we state a conditional existence theorem. It is important to note that the nonlinear dependence of the coefficients of the boundary condition and the conjunction condition on the first derivatives of the unknown function does not permit the use of the Leray-Schauder topological principle (fixed point theorem). Instead we use the Caccioppoli principle (see [7, §1, 5, Chapt. X]) which enables us to circumvent this difficulty thanks due to the exact a priori estimates for the linear problem (I) derived above.

We define

$$
\wp=\left\{(x, t, u, p) \mid(x, t) \in \bar{Q}_{T}, u \in \mathbb{R}, p \in \mathbb{R}^{n}\right\} .
$$

Assumptions II. (1) Uniform parabolicity:

$$
\sum_{i, j=1}^{m} a_{i, j}^{(q)}(x, t, u, p) \xi_{i} \xi_{j} \geq \nu^{(q)}(|u|,|p|) \sum_{i=1}^{m} \xi_{i}^{2}, \quad \forall(x, t, u, p) \in \wp, q=1,2,
$$

where $\nu^{(q)}\left(t_{1}, t_{2}\right)$ are positive continuous functions which are nonincreasing with respect to both arguments and are defined for $t_{1} \geq 0, t_{2} \geq 0$. 
(2) Smoothness: The functions

$$
\begin{aligned}
& a_{i, j}^{(q)}(x, t, u, p), \frac{\partial a_{i, j}^{(q)}(x, t, u, p)}{\partial p_{k}}, \frac{\partial a_{i, j}^{(q)}(x, t, u, p)}{\partial u}, a^{(q)}(x, t, u, p), \\
& \frac{\partial a^{(q)}(x, t, u, p)}{\partial p_{i}}, \frac{\partial a^{(q)}(x, t, u, p)}{\partial u}, b^{(q)}(x, t, u, p), \frac{\partial b^{(q)}(x, t, u, p)}{\partial p_{i}}, \\
& \frac{\partial b^{(q)}(x, t, u, p)}{\partial u}, \frac{\partial^{2} b^{(q)}(x, t, u, p)}{\partial p_{i} \partial p_{j}}, \frac{\partial^{2} b^{(q)}(x, t, u, p)}{\partial u \partial p_{j}}, \\
& \frac{\partial^{2} b^{(q)}(x, t, u, p)}{\partial u \partial x_{j}}, \frac{\partial^{2} b^{(q)}(x, t, u, p)}{\partial u^{2}}, \frac{\partial^{2} b^{(q)}(x, t, u, p)}{\partial p_{i} \partial x_{j}}, b_{i}^{(q)}(x, t, u), \\
& \frac{\partial b_{i}^{(q)}(x, t, u)}{\partial x_{i}}, \frac{\partial b_{i}^{(q)}(x, t, u)}{\partial u}, \frac{\partial^{2} b_{i}^{(q)}(x, t, u)}{\partial u^{2}}, \frac{\partial^{2} b_{i}^{(q)}(x, t, u)}{\partial u \partial x_{i}}, b_{0}^{(q)}(x, t, u), \\
& \frac{\partial^{2} b_{0}^{(q)}(x, t, u)}{\partial u^{2}}, \frac{\partial^{2} b_{0}^{(q)}(x, t, u)}{\partial u \partial x_{j}}, c(x, t, u, p), \frac{\partial c(x, t, u, p)}{\partial p_{i}}, \frac{\partial c(x, t, u, p)}{\partial u}, \\
& \frac{\partial^{2} c(x, t, u, p)}{\partial p_{i} \partial p_{j}}, \frac{\partial^{2} c(x, t, u, p)}{\partial u \partial p_{j}}, \frac{\partial^{2} c(x, t, u, p)}{\partial u \partial x_{j}}, \frac{\partial^{2} c(x, t, u, p)}{\partial u^{2}}, \\
& \frac{\partial^{2} c(x, t, u, p)}{\partial p_{i} \partial x_{j}}, c_{i}(x, t, u), \frac{\partial c_{i}(x, t, u)}{\partial x_{i}}, \frac{\partial c_{i}(x, t, u)}{\partial u}, \\
& \frac{\partial^{2} c_{i}(x, t, u)}{\partial u^{2}}, \frac{\partial^{2} c_{i}(x, t, u)}{\partial u \partial x_{i}}, c_{0}(x, t, u), \frac{\partial^{2} c_{0}(x, t, u)}{\partial u^{2}}, \frac{\partial^{2} c_{0}(x, t, u)}{\partial u \partial x_{j}}, \\
& i, j=1, \ldots, m, \\
& q=1,2,
\end{aligned}
$$

are defined on $\wp$ and have the following properties:

(i) they are bounded;

(ii) they satisfy a Hölder condition with exponent $\lambda \in(0,1)$ with respect to $x_{i}, i=1, \ldots, m$, and with exponent $\lambda / 2$ with respect to $t$;

(iii) they satisfy a Lipschitz condition with respect to $u, p_{i}, i=1, \ldots, m$;

(iv) the functions

$$
\begin{aligned}
& \frac{\partial b^{(q)}(x, t, u, p)}{\partial p_{i}}, \frac{\partial b^{(q)}(x, t, u, p)}{\partial u}, b_{i}^{(q)}(x, t, u), \frac{\partial b_{i}^{(q)}(x, t, u)}{\partial u}, \frac{\partial b_{0}^{(q)}(x, t, u)}{\partial u}, \\
& \frac{\partial c(x, t, u, p)}{\partial p_{i}}, \frac{\partial c(x, t, u, p)}{\partial u}, c_{i}(x, t, u), \frac{\partial c_{i}(x, t, u)}{\partial u}, \frac{\partial c_{0}(x, t, u)}{\partial u}
\end{aligned}
$$

satisfy a Hölder condition with exponent $\frac{1+\lambda}{2}$ with respect to $t$;

$$
\begin{array}{ll}
\psi(x, t) \in C^{(1, \lambda)}\left(\gamma_{T}\right), & \varphi(x, t) \in C^{(1, \lambda)}\left(\partial_{T} \Omega\right), \\
\chi(x, t) \in C^{(2, \lambda)}\left(\gamma_{T}\right), & \omega(x) \in \mathbf{C}^{(2, \lambda)}(\bar{\Omega}) .
\end{array}
$$


(3) Complementarity (see [8]):

$$
\begin{gathered}
\sum_{i=1}^{m}\left\{\frac{\partial b^{(q)}(x, t, u, p)}{\partial p_{i}}+b_{i}^{(q)}(x, t, u)\right\} \gamma_{i}(x) \geq \nu_{0}^{(q)}(|u|,|p|)>0, \\
\forall(x, t) \in \gamma_{T}, u \in \mathbb{R}, p \in \mathbb{R}^{n}, q=1,2, \\
\sum_{i=1}^{m}\left\{\frac{\partial c(x, t, u, p)}{\partial p_{i}}+c_{i}(x, t, u)\right\} n_{i}(x) \geq \nu_{0}(|u|,|p|)>0, \\
\forall(x, t) \in \partial_{T} \Omega, u \in \mathbb{R}, p \in \mathbb{R}^{n},
\end{gathered}
$$

where $\nu_{0}^{(q)}\left(t_{1}, t_{2}\right), \nu_{0}\left(t_{1}, t_{2}\right)$ are positive continuous functions which are nonincreasing with respect to both arguments and are defined for $t_{1} \geq 0, t_{2} \geq 0$.

(4) Compatibility (zero-order compatibility conditions for initial and boundary data, see [8]):

$$
\begin{aligned}
& {[\omega(x)]_{\gamma}=\chi(x, 0), \quad x \in \gamma,} \\
& {\left[\sum_{i, j=1}^{m} a_{i, j}\left(x, 0, \omega, \omega_{x}\right) \omega_{x_{i} x_{j}}(x)+a\left(x, 0, \omega, \omega_{x}\right)\right]_{\gamma}=\frac{\partial \chi(x, 0)}{\partial t}, \quad x \in \gamma,} \\
& {\left[b\left(x, 0, \omega, \omega_{x}\right)+\sum_{i=1}^{m} b_{i}(x, 0, \omega) \omega_{x_{i}}+b_{0}(x, 0, \omega)\right]_{\gamma}=\psi(x, 0), \quad x \in \gamma,} \\
& c\left(x, 0, \omega^{(2)}, \omega_{x}^{(2)}\right)+\sum_{i=1}^{m} c_{i}\left(x, 0, \omega^{(2)}\right) \omega_{x_{i}}^{(2)}+c_{0}\left(x, 0, \omega^{(2)}\right)=\varphi(x, 0), \quad x \in \partial \Omega .
\end{aligned}
$$

(5) $\Omega^{(q)} \in A^{(2, \lambda)}, q=1,2$.

THEOREM 4.1 (Conditional theorem). Suppose that four families of differential operators $L_{(\tau)}^{(q)}\left(x, t, D_{x}\right), q=1,2, \Lambda_{(\tau)}^{\gamma_{T}}, \Lambda_{(\tau)}^{\partial_{T} \Omega}$ are as in (III), continuously depend on the parametr $\tau \in[0,1]$, satisfy assumptions (1)-(5) uniformly with respect to $\tau \in[0,1]$ and are such that for $\tau=1$ the problem

$\left(\mathrm{III}_{\tau}\right)$

$$
\left\{\begin{array}{l}
L_{(\tau)}^{(q)}\left(x, t, D_{x}\right) u^{(q)}=0, \quad(x, t) \in Q_{T}^{(q)}, q=1,2, \\
{[u]_{\gamma_{T}}=\chi(x, t),} \\
{\left[\Lambda_{(\tau)}^{\gamma_{T}} u\right]=\psi(x, t), \quad(x, t) \in \gamma_{T},} \\
\Lambda_{(\tau)}^{\partial_{T} \Omega} u^{(2)}=\varphi(x, t), \quad(x, t) \in \partial_{T} \Omega, \\
u^{(q)}(x, 0)=\omega^{(q)}(x), \quad x \in \overline{\Omega^{(q)}}, q=1,2,
\end{array}\right.
$$

coincides with (III). Let the following conditions hold:

(a) for all solutions $u(x, t, \tau) \in \mathbf{C}^{(2, \lambda)}\left(\bar{Q}_{T}\right)$ of $\left(\mathrm{III}_{\tau}\right)$ the corresponding variational problem is unconditionally solvable; 
(b) the possible solutions of $\left(\mathrm{III}_{\tau}\right)$ and their first derivatives are uniformly bounded and equicontinuous in $\bar{Q}_{T}$ for all $\tau \in[0,1]$ as $\chi(x, t), \psi(x, t)$, $\varphi(x, t), \omega(x)$ vary in all families of functions for which the corresponding quantities $\Xi_{0}+\Xi_{2, \lambda}, \Psi_{0}+\Psi_{1, \lambda}, \Phi_{0}+\Phi_{1, \lambda}, \Omega_{0}+\Omega_{2, \lambda}$ remain bounded;

(c) for $\tau=0$ and fixed $\psi(x, t) \in C^{(1, \lambda)}\left(\gamma_{T}\right), \varphi(x, t) \in C^{(1, \lambda)}\left(\partial_{T} \Omega\right)$, $\chi(x, t) \in C^{(2, \lambda)}\left(\gamma_{T}\right), \omega(x) \in \mathbf{C}^{(2, \lambda)}(\bar{\Omega})$ there exists a unique solution $u(x, t)$ $\in \mathbf{C}^{(2, \lambda)}\left(\bar{Q}_{T}\right)$ of $\left(\mathrm{III}_{\tau}\right)$.

Then for any $\psi(x, t) \in C^{(1, \lambda)}\left(\gamma_{T}\right), \varphi(x, t) \in C^{(1, \lambda)}\left(\partial_{T} \Omega\right), \chi(x, t) \in$ $C^{(2, \lambda)}\left(\gamma_{T}\right), \omega(x) \in \mathbf{C}^{(2, \lambda)}(\bar{\Omega})$ there exists a unique solution $u(x, t) \in$ $\mathbf{C}^{(2, \lambda)}\left(\bar{Q}_{T}\right)$ of $\left(\mathrm{III}_{\tau}\right)$, for every $\tau \in[0,1]$.

This theorem is proved in the same way as in $[7, \S 42]$; [5, Chapt. X]; [3]; $[1, \S 3]$ on the basis of the exact a priori estimate (1.33).

\section{References}

[1] M. V. Borsuk, A priori estimates and solvability of second order quasilinear elliptic equations in a composite domain with nonlinear boundary conditions and a conjunction condition, Proc. Steklov Inst. Math. 103 (1970), 13-51.

[2] - - An exact a priori estimate of Schauder type for solutions of the oblique derivative problem for a linear second order parabolic equation with discontinuous coefficients, Dokl. Akad. Nauk Ukrain. SSR. Ser. A 1986, no. 6, 3-5 (in Russian).

[3] R. Fiorenza Sui problemi di derivata obliqua per le equazioni ellittiche, Ricerche Mat. 8 (1959), 83-110.

[4] L. I. Kamynin and V. N. Maslennikova, Boundary estimates of solutions of the third boundary value problem for a parabolic equation, Dokl. Akad. Nauk SSSR 153 (1963), 526-529 (in Russian).

[5] O. A. Ladyzhenskaya and N. N. Ural'tseva, Linear and Quasilinear Equations of Elliptic Type, Academic Press, New York, 1968.

[6] O. A. Ladyzhenskaya, N. N. Ural'tseva and V. A. Solonnikov, Linear and Quasilinear Equations of Parabolic Type, Nauka, Moscow, 1967 (in Russian).

[7] C. Miranda, Partial Differential Equations of Elliptic Type, 2nd ed., Springer, New York, 1970.

[8] N. V. Zhitarashu, Estimates of Schauder type and solvability of general boundary value problems for general parabolic systems with discontinuous coefficients, Dokl. Akad. Nauk SSSR 169 (1966), 511-514 (in Russian).

Department of Mathematics and Informatics

University of Warmia and Mazury

Licznerskiego 4

10-721 Olsztyn, Poland

E-mail: borsuk@uwm.edu.pl 